\title{
Bosonization, Topological Solitons and Fractional Charges in Two-Dimensional Quantum Field Theory
}

\author{
Jürg Fröhlich ${ }^{1}$ and Pieralberto Marchetti ${ }^{2}$ \\ ${ }^{1}$ Theoretical Physics, ETH-Hönggerberg, CH-8093 Zürich, Switzerland \\ ${ }^{2}$ Dipartimento di Fisica, Universita di Padova, I.N.F.N., I-35131 Padova, Italy
}

\begin{abstract}
We further develop the quantization of topological solitons in twodimensional quantum field theory in terms of Euclidean region functional integrals. Our approach is nonperturbative and mathematically rigorous. We apply it to construct physical states with fractional fermion number in models of interacting bosons and fermions without recurring to a semiclassical approximation. A related issue discussed in this paper is two-dimensional chiral bosonization.
\end{abstract}

\section{Introduction}

In this paper we reconsider the quantum theory of solitons in quantum field models in two space-time dimensions. Our purpose is to construct Euclidean Green functions of local order fields and soliton fields in terms of Euclidean region functional integrals. We also discuss the algebraic structure defined by the orderand the soliton fields [1] and associated monodromy structure of their Euclidean Green functions. It will turn out that those Green functions are simply correlation functions of products of order- and disorder variables.

A mathematically rigorous approach to soliton quantization within the Hamiltonian formalism of quantum field theory was first developed in [1]. It turned out, however, that for purposes of a detailed analysis of concrete models a Euclidean approach to soliton quantization would be more powerful; (see [2] for a systematic treatment of soliton quantization in terms of Euclidean region functional integrals within the context of lattice theory).

The idea that the superselection structure of a large class of quantum field theories in two and more space-time dimensions is encoded, in a mathematically precise sense, in the set of correlation functions of general local order- and disorder variables (especially disorder variables associated with line defects of the corresponding Euclidean field theory) was first conceived in the first reference quoted in [3], although of course order-disorder correlation functions had already been studied earlier, and attempts had been made to extract information about the field theory from such order-disorder correlation functions [4]. The proposal in 
[3a] was also made, independently, in [3b] and systematized in $[2,3]$. In this paper, we combine results and methods from constructive field theory, $[5,6]$ with the ideas in $[2,3]$ to develop a systematic, non-perturbative and mathematically rigorous approach to soliton quantization for quantum field models in two spacetime dimensions. Our approach is based on constructing modified Euclidean region functional integrals in terms of which the soliton Green functions can be calculated. The modified functional integrals arise by coupling the basic Euclidean region fields of a field theory to singular external gauge fields whose curvature is concentrated in a finite set of points in Euclidean space-time where soliton fields are inserted. The gauge group relevant for our construction is simply the global symmetry group of the classical action, and the coupling of the basic Euclidean fields of the theory to the external gauge field is accomplished by "minimal substitution," i.e. by replacing derivatives by covariant derivatives. In this way, the basic Euclidean fields of the theory turn into distributional sections of some vector bundle over punctured Euclidean space-time, with those space-time points deleted where a soliton field is inserted. This vector bundle is equipped with a flat connection with nontrivial holonomy.

One might object that the time for such an analysis of quantum solitons has passed. However, the ideas and methods that we develop have several applications to more topical problems of present-day quantum field theory. Here are some examples: We present a formalism for bosonizing arbitrary Euclidean Green functions of chiral spin-1/2 Fermi fields and their local currents in two space-time dimensions. We are able to express such correlation functions in terms of bona fide Gaussian integrals of products of functionals of a real, free, massless Bose field and of disorder operators for that field. In principle, our approach extends to arbitrary Riemann surfaces, but we have not bothered to work out the details. While at the level of identities between Bose- and Fermi Green functions we have no new results to report, the fact that Green functions of chiral Fermi fields can be expressed in terms of bona fide bosonic functional integrals may have escaped attention.

We are also able to express correlation functions of spin fields associated with two-dimensional Dirac fermions in terms of Gaussian integrals of products of functionals of a free, massless Bose field. By "taking square roots" a similar result is obtained for the correlation functions of spin fields associated with a twodimensional Majorana fermion. This is of interest in connection with the covariant quantization of the superstring, in particular with deriving formulas for fermion emission vertices. But it also yields explicit formulae for the spin correlations of the scaling limit of the two-dimensional, critical Ising model. This is, of course, not a new result. But our approach to bosonization could be used to analyze several other two-dimensional, critical models of statistical mechanics wich can be "mapped" onto the Gaussian model; (critical XY-and clock models, Ashkin-Teller model, ...). It is also of some interest in connection with some monodromy representations of the braid groups, (the Burau representations). However, in this paper there is no room for a systematic study of these matters.

Finally, our approach to soliton quantization can be used for a nonperturbative, mathematically rigorous analysis of kinks and antikinks carrying fractional fermion numbers in models of interacting bosons and fermions, such as the pseudo-scalar Yukawa model, in two space-time dimensions. Our analysis 
goes beyond the semiclassical approximation and replaces topological arguments applied in conjunction with the semiclassical approximation by algebra.

It is worthwhile mentioning that an attempt to evaluate our non-perturbative formulas for solition Green functions quantitatively, with the help of a semiclassical expansion, leads to some intriguing classical variational problems which we know how to deal with only in the simplest cases.

The organization of our paper is as follows:

In Sect. 2, we discuss the quantization of solitons in terms of functional integrals for the simple example of the $\lambda \phi_{2}^{4}$ model. We derive non-perturbative expressions for mixed order field-soliton Euclidean Green functions and recover the "dual algebra" commutation relations [1] between order fields and soliton fields. We show how the $\lambda \phi_{2}^{4}$ model can be reformulated as a theory of an interacting Majorana field. It is worth mentioning here that there is a related, mathematically rigorous isomorphism between the $\lambda \phi^{4}$ model in three space-time dimensions and a nontrivial $\mathbb{Z}_{2}$-gauge theory. These isomorphisms extend the Onsager transformation and Kramers-Wannier duality from the Ising- to the $\lambda \phi^{4}$ models. Finally, we comment on the semiclassical expansion of mixed order fieldsoliton Green functions and formulate an associated classical variational problem.

In Sect. 3, we review chiral bosonization over the complex plane (isomorphic to two-dimensional Euclidean space-time) in a form that we believe is somewhat novel. We express spin fields associated with Dirac fermions as functionals of a free, massless Bose field and sketch how one may use this to calculate the correlation functions of the two-dimensional, critical Ising model in the scaling limit.

In Sect. 4, we briefly review the Euclidean quantization of solitons in the twodimensional sine-Gordon model and show how to reconstruct charged Fermi fields out of our local soliton fields.

In Sect. 5, we combine the results of Sects. 2 through 4 to carry out a nonperturbative analysis of fractional fermion numbers in two-dimensional models of interacting bosons and fermions. We hope that our analysis may clarify some aspects of this phenomenon which, as is well known, is of intereset in onedimensional condensed matter physics.

In an appendix some features of the lattice approximation to the bosonized, two-dimensional pseudo-scalar Yukawa model are briefly discussed.

Fairly detailed references to previous work on all these problems may be found in Sects. 2 through 5.

In a companion paper we shall analyze the Euclidean quantization of "anyons," i.e. point vortices carrying a fractional electric charge, in threedimensional Higgs models with Chern-Simons term in the action. Our analysis which is completely nonperturbative sheds some light on strange properties of anyons, like their fractional spin and statistics, their fractional electric charge and their "extended particle" structure.

\section{A Simple Model with Solitons: $\lambda \phi_{2}^{4}$ Theory}

In this section we describe the construction of soliton-, or kink Euclidean Green functions in the simple $\lambda \phi_{2}^{4}$ model and show that this theory can also be formulated 
as a theory of Majorana fermions. The formalism introduced here will be useful in our discussion of kinks carrying fractional fermion number in two-dimensional models of interacting bosons and fermions (Sect. 5) and is therefore developed in some detail.

To begin with, we briefly recall the kink solutions of the classical $\lambda \phi_{2}^{4}$ field theory. The Hamiltonian of the model is

$$
H=\int_{-\infty}^{\infty} d x \frac{1}{2}\left[\pi(x)^{2}+(\nabla \phi)^{2}(x)+\frac{\lambda}{2}\left(\phi(x)^{2}-\xi\right)^{2}\right] .
$$

The absolute minima of $H$ in the infinite-dimensional phase space of the system are

$$
\phi(x)= \pm \sqrt{\xi}, \quad \pi(x)=0, \quad \text { (independent of } x \text { ). }
$$

The second functional derivative of $H$ in $\phi$ at these points is

$$
\frac{\delta^{2} H}{\delta \phi(x) \delta \phi(y)}(\phi(x)= \pm \sqrt{\xi}, \pi=0)=\left(-\partial_{x}^{2}+m^{2}\right) \delta(x-y),
$$

where the mass $m$ is given by

$$
m^{2}= \begin{cases}2 \lambda \xi, & \xi>0 \\ -\lambda \xi, & \xi<0 .\end{cases}
$$

Every configuration $(\pi(x), \phi(x))$ of finite energy satisfies

$$
\lim _{x \rightarrow \pm \infty} \pi(x)=0, \quad \lim _{x \rightarrow \infty} \phi(x)= \pm \sqrt{\xi}, \quad \lim _{x \rightarrow-\infty} \phi(x)= \pm \sqrt{\xi} .
$$

Thus the space of finite-energy configurations consists of four disconnected components

$$
\begin{gathered}
\Gamma_{+}=\left\{(\pi, \phi): \lim _{x \rightarrow \pm \infty} \phi(x)=\sqrt{\xi}\right\}, \\
\Gamma_{-}=\left\{(\pi, \phi): \lim _{x \rightarrow \pm \infty} \phi(x)=-\sqrt{\xi}\right\}, \\
\Gamma_{s}=\left\{(\pi, \phi): \lim _{x \rightarrow+\infty} \phi(x)=-\lim _{x \rightarrow-\infty} \phi(x)=\sqrt{\xi}\right\},
\end{gathered}
$$

and

$$
\Gamma_{\bar{s}}=\left\{(\pi, \phi): \lim _{x \rightarrow+\infty} \phi(x)=-\lim _{x \rightarrow-\infty} \phi(x)=-\sqrt{\xi}\right\} .
$$

The minima of the Hamiltonian $H$ restricted to $\Gamma_{s}$ and $\Gamma_{\bar{s}}$ give rise to kink- and anti-kink solutions, $\phi_{s}(x), \phi_{\bar{s}}(x)=-\phi_{s}(x)$, of the classical field equations. Here

$$
\phi_{s}(x)=\sqrt{\xi} \tanh \left(\sqrt{\frac{\lambda \xi}{2}} x-a\right)=-\phi_{\bar{s}}(x),
$$

for arbitrary real $a$.

Travelling kinks or anti-kinks are obtained by applying Lorentz boosts to the solutions (2.6). 
Fig. 1

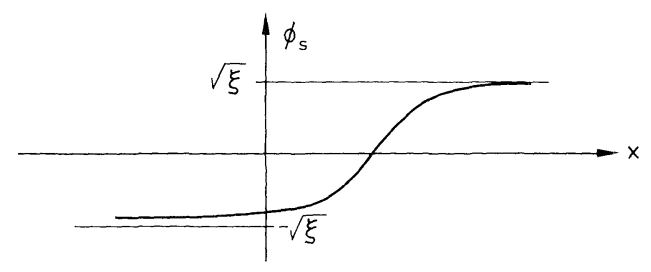

The components $\Gamma_{+}, \Gamma_{-}, \Gamma_{s}$, and $\Gamma_{\bar{s}}$ are invariant under the Hamiltonian flow. This permits us to associate a time-independent topological charge $Q(\phi)$ with every finite-energy solution, $\phi(t, x)$, of the classical field equations,

$$
Q(\phi)=\int_{-\infty}^{+\infty} \phi_{, x}(t, x) d x=\lim _{x \rightarrow \infty}[\phi(t, x)-\phi(t,-x)] .
$$

For $(\dot{\phi}, \phi) \in \Gamma_{ \pm}$, we find $Q(\phi)=0$, for $(\dot{\phi}, \phi) \in \Gamma_{s}, Q(\phi)=2 \sqrt{\xi}$, and for $(\dot{\phi}, \phi) \in \Gamma_{\bar{s}}$, $Q(\phi)=-2 \sqrt{\xi}$.

\subsection{Construction of the Vacuum Sector}

We now pass to the quantum theory of kinks and anti-kinks in the $\lambda \phi_{2}^{4}$ model. The Hamiltonian approach to this problem has been investigated rigorously and in much detail in [1]. Here we briefly review the quantization of kinks and anti-kinks in terms of Euclidean region functional integrals, as developed in [2]. (For earlier work in this direction, see also [3].) In the Euclidean description of quantum field theory, the $\lambda \phi_{2}^{4}$ model is characterized by its Euclidean action

$$
S(\Phi)=\int d^{2} x\left\{\frac{1}{2}:(\nabla \Phi)^{2}:(x)+\frac{\lambda}{4}:\left(\Phi^{2}-\xi\right)^{2}:(x)\right\},
$$

where $\Phi(x)$ is a random field whose distribution is given by the probability measure

$$
d \mu_{ \pm}(\Phi)=\frac{1}{Z} e^{-S(\Phi)} D \Phi_{ \pm}
$$

In (2.8), the double dots indicate normal ordering with respect to the free propagator $\left(-\nabla+m^{2}\right)^{-1}$, where $m^{2}$ is given by (2.3). Formula (2.9) is the Euclidean Gell-Mann-Low formula: $D \Phi_{ \pm}$is a formal Lebesgue measure on $\times \mathbb{R}_{x}$, and the subscript " \pm " indicates that we impose boundary conditions (b.c.)

$$
\Phi(x) \rightarrow+\sqrt{\xi}, \quad \text { as } \quad|x| \rightarrow \infty, \quad \text { or } \quad \Phi(x) \rightarrow-\sqrt{\xi}, \quad \text { as }|x| \rightarrow \infty,
$$

for $\xi>0$. (For $\xi<0$ we impose zero Dirichlet b.c., $\Phi(x) \rightarrow 0$, as $|x| \rightarrow \infty$.) The precise mathematical definition of these b.c. is discussed in $[5,6]$; [the notation $(2.10)$ is somewhat symbolic]. The b.c. (2.10) are chosen so as to select pure phases of the theory.

The factor $1 / Z$ is chosen such that $d \mu_{+}(\Phi)$ and $d \mu_{-}(\Phi)$ are probability measures on the function space $\mathscr{S}^{\prime}\left(\mathbb{R}^{2}\right)$ of tempered distributions on $\mathbb{R}^{2}$. Formula (2.9) has been given a rigorous mathematical interpretation; see e.g. $[5,6]$. For example, $d \mu_{+}(\Phi)$ and $d \mu_{-}(\Phi)$ can be viewed as limits of measures describing fields over a 
bounded space-(imaginary) time lattice, as the lattice spacing tends to 0 and the thermodynamic (infinite-volume) limit is taken. It is known that, for $\xi$ small enough,

$$
d \mu_{+}(\Phi)=d \mu_{-}(\Phi) \equiv d \mu(\Phi)
$$

while for $\xi>\xi_{c}$, for some bounded $\xi_{c}$,

$$
d \mu_{+}(\Phi)=d \mu_{-}(-\Phi) \neq d \mu_{-}(\Phi)
$$

This is interpreted, physically, as spontaneous symmetry breaking: The action $S(\Phi)$ defined in (2.8) and the formal Lebesgue measure have a discrete, global $\mathbb{Z}_{2}$ symmetry

$$
\Phi(x) \rightarrow-\Phi(x), \text { for all } x \in \mathbb{R}^{2} .
$$

For $\xi$ sufficiently large, this symmetry is spontaneously broken, and the boundary conditions (2.10) select two distinct pure phases characterized by $d \mu_{+}(\Phi), d \mu_{-}(\Phi)$, respectively, [5b]. This is expected on the basis of the classical theory; see (2.2).

The Euclidean Green functions of the $\lambda \phi_{2}^{4}$ model are the moments of $d \mu_{ \pm}$, i.e.

$$
G_{ \pm}^{(n)}\left(x_{1}, \ldots, x_{n}\right)=\int \prod_{i=1}^{n} \Phi\left(x_{i}\right) d \mu_{ \pm}(\Phi) .
$$

It is known that these Green functions satisfy the Osterwalder-Schrader axioms [7]; (distribution property, Euclidean invariance, clustering and OsterwalderSchrader-, or reflection positivity). The Osterwalder-Schrader reconstruction theorem then guarantees that the $\left\{G_{ \pm}^{(n)}\right\}$ uniquely determine real-time Wightman distributions and Green functions by analytic continuation in the time variables. If

$$
x_{1}^{0}<x_{2}^{0}<\ldots<x_{n}^{0}, \quad x \equiv\left(x^{0}, x^{1}\right),
$$

then

$$
G_{ \pm}^{(n)}\left(x_{1}, \ldots, x_{n}\right)=\left\langle\Omega_{ \pm}, \phi\left(x_{1}^{1}\right) e^{-\left(x_{2}^{0}-x_{1}^{0}\right) H} \phi\left(x_{2}^{1}\right) \ldots e^{-\left(x_{n}^{0}-x_{n-1}^{0}\right) H} \phi\left(x_{n}^{1}\right) \Omega_{ \pm}\right\rangle,
$$

where $\Omega_{+}$and $\Omega_{-}$are the physical vacua (distinct, for $\xi>\xi_{c}$, but $\Omega_{+}=\Omega_{-} \equiv \Omega$, for $\xi$ small enough), $\phi\left(x^{1}\right)$ is the relativistic time- 0 quantum field, and $H$ is the Hamilton operator of the $\lambda \phi_{2}^{4}$ model.

For $\xi>\xi_{c}$,

$$
\left\langle\Omega_{+}, \phi(x) \Omega_{+}\right\rangle=-\left\langle\Omega_{-}, \phi(x) \Omega_{-}\right\rangle=\Phi_{c}>0,
$$

while, for $\xi$ small enough, $\langle\Omega, \phi(x) \Omega\rangle=0$.

Let $\mathscr{F}_{+}$be the algebra of functionals of the Euclidean field $\Phi$ generated by

$$
\left\{\Phi(f): f \in \mathscr{S}\left(\mathbb{R}^{2}\right), \operatorname{supp} f \subseteq\left\{\left(x^{0}, x^{1}\right): x^{0}>0\right\}\right\} .
$$

We define

$$
f_{\theta}\left(x^{0}, x^{1}\right)=f\left(-x^{0}, x^{1}\right), \quad f_{t}\left(x^{0}, x^{1}\right)=f\left(x^{0}-t, x^{1}\right)
$$

and

$$
f_{a}\left(x^{0}, x^{1}\right)=f\left(x^{0}, x^{1}-a\right)
$$


Furthermore

$$
\Phi_{\theta}(f)=\Phi\left(f_{\theta}\right), \quad \Phi_{t}(f)=\Phi\left(f_{t}\right), \quad \text { and } \quad \Phi_{a}(f)=\Phi\left(f_{a}\right) .
$$

For $F \in \mathscr{F}_{+}$, we define

$$
\theta F(\Phi)=F\left(\Phi_{\theta}\right), \quad F_{t}(\Phi)=F\left(\Phi_{t}\right), \quad \text { and } \quad F_{a}(\Phi)=F\left(\Phi_{a}\right) .
$$

The image of $\mathscr{F}_{+}$under $\theta$ is denoted $\mathscr{F}_{-}$. It is the algebra of functionals of $\Phi$ supported at negative (imaginary) time. For $F \in \mathscr{F}_{+}, t>0, a$ real, $F_{t}$ and $F_{a}$ belong to $\mathscr{F}_{+}$.

Reflection positivity is the property of $d \mu_{ \pm}$that

$$
\langle\overline{\theta F} F\rangle_{ \pm} \geqq 0 \text {, for all } F \in \mathscr{F}_{+},
$$

where

$$
\langle F\rangle_{ \pm} \equiv \int_{\mathscr{S}^{\prime}} F(\Phi) d \mu_{ \pm}(\Phi) .
$$

Reflection positivity permits us to reconstruct the vacuum sectors of the model as follows: With each $F \in \mathscr{F}_{+}$we associate a vector $|F\rangle$ in a Hilbert space $\mathscr{H}_{ \pm}$with scalar product given by

$$
\langle F \mid G\rangle \equiv\langle\overline{\theta F} G\rangle_{ \pm} .
$$

The energy-momentum operator $(H, P)$ is obtained from the formulae

$$
\left\langle F\left|e^{-t H}\right| G\right\rangle \equiv\left\langle F \mid G_{t}\right\rangle=\left\langle\overrightarrow{\theta F} G_{t}\right\rangle_{ \pm},
$$

and

$$
\left\langle F\left|e^{i a P}\right| G\right\rangle \equiv\left\langle F \mid G_{a}\right\rangle=\left\langle\overline{\theta F} G_{a}\right\rangle_{ \pm} .
$$

Similarly, if the measures $d \mu_{ \pm}(\Phi)$ are Euclidean invariant a unitary representation of the full quantum-mechanical Poincaré group on the Hilbert spaces $\mathscr{H}_{+}, \mathscr{H}_{-}$can be reconstructed from the expectation vaiues of products $\overline{\theta F} G$, with $F$ and $G$ in $\mathscr{F}_{+}$, in the measures $d \mu_{+}, d \mu_{-}$, respectively. This is explained in detail in [8].

Finally, the quantum field $\phi$ is obtained from the formulae

$$
\begin{gathered}
\left\langle F\left|\phi\left(0, x^{1}\right)\right| G\right\rangle=\left\langle\overline{\theta F} \Phi\left(0, x^{1}\right) G\right\rangle_{ \pm}, \\
\left\langle F\left|\phi\left(0, x^{1}\right) e^{-t H} \phi\left(0, y^{1}\right)\right| G\right\rangle=\left\langle\overline{\theta F} \Phi\left(0, x^{1}\right) \Phi\left(t, y^{1}\right) G_{t}\right\rangle_{ \pm},
\end{gathered}
$$

etc.

For the $\lambda \phi_{2}^{4}$ model, it is known that, for $|\xi|$ sufficiently large, the spectrum of the mass operator $M$, where $M^{2}=H^{2}-P^{2}$, consists of $\{0\} \cup\left\{m_{\text {phys }}\right\} \cup\left[m_{\text {phys }}+\mu, \infty\right)$, $m_{\text {phys }}>0$ and $\mu>0$. Here $m_{\text {phys }}$ is the mass gap, and $\mu$ is the upper gap. See [5a] for a review and references to the original papers.

The material reviewed between (2.15) and (2.22) is, of course, modelindependent. Similar considerations apply to all other models discussed in subsequent sections of this paper. See $[2,8]$ for general discussions of Osterwalder-Schrader reconstruction [7] in a context adequate for the present paper.

We remark that all results for the $\lambda \phi_{2}^{4}$ model reviewed here are well known, rigorous results. It is quite straightforward to verify them for the lattice model, 
whose Euclidean vacuum functional measure is given by

$$
d \mu_{ \pm}(\Phi)=\lim _{\Lambda^{\prime} \mathbb{Z}^{2}} \frac{1}{Z_{\Lambda}} \exp \left[\sum_{\langle i j\rangle \cap \Lambda \neq \emptyset}\left(\Phi_{i}-\Phi_{j}\right)^{2}\right] \prod_{j \in \Lambda} d \varrho\left(\Phi_{j}\right),
$$

where $\Lambda$ is a finite subset of $\mathbb{Z}^{2}, Z_{A}$ is the partition function,

$$
d \varrho(\Phi)=\exp \left[-\frac{\lambda}{4}\left(\Phi^{2}-\xi\right)^{2}\right] d \Phi,
$$

and

$$
\Phi_{j}= \pm \sqrt{\xi}, \text { for all } j \notin \Lambda \text {. }
$$

In this context, symmetry breaking for $\xi_{>} \xi_{c}\left(\xi_{c}=\xi_{c}(\lambda)>0\right)$ is an easy consequence of a Peierls argument. See e.g. [2, 5a, 6].

The point is now that in the phase of the $\lambda \phi_{2}^{4}$ model with a broken $\phi \rightarrow-\phi$ symmetry the physical state space is much larger than the vacuum sector $\mathscr{H}_{+}$, or $\mathscr{H}_{-},[1]$. There are physical states, $|s\rangle$ of finite total energy with the property that the function $\left\langle s\left|\phi\left(x^{0}, x^{1}\right)\right| s\right\rangle$ resembles the soliton solution $\phi_{s}\left(x^{0}, x^{1}\right)$ of the classical field equations. In particular,

$$
\lim _{x^{1 \rightarrow \pm \infty}}\left\langle s\left|\phi\left(x^{0}, x^{1}\right)\right| s\right\rangle= \pm \Phi_{c},
$$

where $\Phi_{c}$ is the vacuum expectation value of $\phi$ defined in (2.15). Defining the quantum topological charge $Q$ by

$$
Q=\int_{-\infty}^{+\infty} d x^{1}\left(\frac{\partial}{\partial x^{1}} \phi\right)\left(x^{0}, x^{1}\right)
$$

we conclude from (2.25) that

while

$$
\langle s|Q| s\rangle=2 \Phi_{c}
$$

$$
\langle F|Q| G\rangle=0,
$$

for all $|F\rangle,|G\rangle \in \mathscr{H}_{ \pm}$, since, by (2.22),

$$
\begin{aligned}
\langle F|Q| G\rangle & =\lim _{x^{1 \rightarrow \infty}}\left[\left\langle\theta F \Phi\left(0, x^{1}\right) G\right\rangle_{ \pm}-\left\langle\theta F \Phi\left(0,-x^{1}\right) G\right\rangle_{ \pm}\right] \\
& =\langle\theta F G\rangle_{ \pm} \lim _{x^{1} \rightarrow \infty}\left[\left\langle\Phi\left(0, x^{1}\right)\right\rangle_{ \pm}-\left\langle\Phi\left(0,-x^{1}\right)\right\rangle_{ \pm}\right] \\
& =0
\end{aligned}
$$

by clustering.

Our purpose is now to construct a local field, $s(x)$, carrying a topological charge of $\pm 2 \Phi_{c}$, out of which soliton states $|s\rangle,|\bar{s}\rangle$ of charge $\pm 2 \Phi_{c}$ can be constructed.

\subsection{Soliton Bundles for the $\lambda \phi_{2}^{4}$ Model}

In this section we construct a local soliton field, $s(x)$, for the $\lambda \phi_{2}^{4}$ model and show that, for $\xi>\xi_{c}$, where the $\phi \rightarrow-\phi$ symmetry is spontaneously broken, it couples the 
vacuum sectors to soliton sectors of topological charge $\pm 2 \Phi_{c}$. Our construction is purely Euclidean: We directly construct Euclidean Green functions $G_{s, \pm}^{(2 n)}$ for the soliton field and obtain the soliton sectors $\mathscr{H}_{s}, \mathscr{H}_{\bar{s}}$, a representation of the Poincaré group on $\mathscr{H}_{s}, \mathscr{H}_{\bar{s}}$ and local field operators, $s(x)$, from $G_{s, \pm}^{(2 n)}$ by a version [2] of the Osterwalder-Schrader reconstruction theorem [7, 8]. Our construction of $G_{s, \pm}^{(2 n)}$ is inspired by the one in $[2,3]$ which relies on disorder operators. We just sketch the main ideas; details may be found in [9].

We choose $2 n$ points $\left\{x_{1}, \ldots, x_{2 n}\right\}$ in Euclidean space-time $\mathbb{R}^{2}$ and define

$$
M=\mathbb{R}^{2} \backslash\left\{x_{1}, \ldots, x_{2 n}\right\} .
$$

Clearly, $M$ is not simply connected, and there exist, therefore, non-trivial real line bundles, $E$, over $M$ with fibre $\mathbb{R}$ and structure group $\mathbb{Z}_{2}$. The group action on the fibres is given by

$$
\left(\varepsilon \in \mathbb{Z}_{2}=\{1,-1\}, \phi \in \mathbb{R}\right) \rightarrow \varepsilon \phi .
$$

Such bundles are uniquely characterized by their holonomy: With each point $x_{i}$ we associate a number $q_{i}=0$, or 1 . Let $\omega$ be a loop in $M$. We let $n\left(\omega, x_{i}\right)$ denote the winding number of $\omega$ with respect to the point $x_{i}$. We set

$$
U(\omega)=(-1)^{\sum_{i=1}^{2 n} q_{i} n\left(\omega . x_{i}\right)} .
$$

$U$ defines a representation of the fundamental group of $M$. All line bundles $E$ specified above may be characterized by such a representation, or, in other words, by a choice of $q_{1}, \ldots, q_{2 n}$. From now on we choose

$$
q_{1}=\ldots=q_{2 n}=1 \text {. }
$$

An explicit choice of a flat connection on $E$ can be made by connecting the points $x_{1}, \ldots, x_{2 n}$ pairwise by $n$ paths $\underline{\gamma}=\left(\gamma_{1}, \ldots, \gamma_{n}\right)$ and defining parallel transporters along arbitrary paths $\pi$ in $M$ by

$$
U(\pi, \underline{\gamma})=(-1)^{\sum^{n}=1} i\left(\pi, \gamma_{J}\right)
$$

where

$$
i(\pi, \gamma)=\# \text { intersections of } \pi \text { with } \gamma \text {. }
$$

This permits us to define a covariant derivative, $\nabla_{\gamma}$, and a covariant Laplacian, $\Delta_{\gamma}$, on sections of $E$. For our analysis of the field theory, the only important quantity is the Green function of $\Lambda_{\gamma}$ which can be calculated neatly by using Brownian motion: Let $d W_{x y}^{T}(\pi)$ be the standard Wiener measure on continuous paths, $\pi$, parametrized by the interval $[0, T]$ with $\pi(0)=x, \pi(T)=y,(x, y \in M)$. Then the Green function, $G_{\underline{\gamma}}(x, y)$, of $\Delta_{\underline{\gamma}}$ is given by

$$
G_{y}(x, y)=\int_{0}^{\infty} d T \int d W_{x v}^{T}(\pi) U(\pi, \underline{y}) .
$$

The meaning of $\nabla_{y}$ and $\Delta_{\underline{\gamma}}$ can be elucidated by embedding $E$ in a complex line bundle with structure group $U(1)$ carrying a regular $U(1)$-connection, $A$. One obtains $\nabla_{\gamma}, \Delta_{\gamma}$ from $\nabla_{A}, \Delta_{A}$ by studying the limit where the curvature, $d A$, gets 
concentrated in $x_{1}, \ldots, x_{2 n},(d A)(x) \rightarrow \pi \sum_{j=1}^{2 n} \pm \delta\left(x-x_{j}\right)$, and the support of $A$ shrinks
to $\underline{\gamma}$; see also [9].]

We now define a covariant action by

$$
S(\Phi, \underline{\gamma})=\int d^{2} x\left\{\frac{1}{2}:\left(\nabla_{\gamma} \Phi\right)^{2}:(x)+\frac{\lambda}{4}:\left(\Phi^{2}-\xi\right)^{2}:(x)\right\} .
$$

Given $x \in M$, let $\pi_{x}$ be the path parallel to the $x^{1}$ axis starting at $x$ and reaching out to $x^{1}=+\infty$. We introduce gauge-invariant fields

$$
\Phi_{\underline{\gamma}}(x)=\Phi(x) U\left(\pi_{x}, \underline{\gamma}\right) .
$$

The full set of Green functions of the $\lambda \phi_{2}^{4}$ model is then given by

$$
G_{s, \pm}^{(2 n, m)}\left(x_{1}, \ldots, x_{2 n} ; y_{1}, \ldots, y_{m}\right)=\left[\frac{1}{Z} \int_{\Gamma_{d}(E)} e^{-S(\Phi, \underline{\gamma})} \prod_{j=1}^{m} \Phi_{\underline{\gamma}}\left(y_{j}\right) D_{E} \Phi_{ \pm}\right]_{\mathrm{ren}},
$$

where $\Gamma_{d}(E)$ is a space of distributional sections of $E$, and $D_{E} \Phi^{ \pm}$is the formal Lebesgue measure on $\Gamma_{d}(E)$. The subscripts, \pm , still refer to b.c.

$$
\Phi(x) \rightarrow \pm \sqrt{\xi}, \quad \text { as } \quad|x| \rightarrow \infty,
$$

and $[\ldots]_{\text {ren }}$ indicates that the functional integral between the square brackets requires a multiplicative renormalization of the form $\alpha(\kappa)^{2 n}$, where $\alpha(\kappa) \rightarrow \infty$, as an ultraviolet regularization $\kappa$ is removed, (e.g. the lattice spacing of a lattice $\lambda \phi_{2}^{4}$ model is sent to zero). It is not really hard to give (2.36) a rigorous mathematical meaning; see [9]. In fact, the main problems to resolve are to define the Gaussian measure $d \mu_{\gamma}^{0}(\Phi)$ with mean 0 and covariance $\left(-\Delta_{\gamma}+m^{2}\right)^{-1}, m^{2}=2 \lambda \xi(\xi>0)$ on $\Gamma_{d}(E)$ - which is straightforward, using

$$
\left(-\Delta_{\gamma}+m^{2}\right)^{-1}(x, y)=\int_{0}^{\infty} d T e^{-m^{2} T} \int d W_{x y}^{T}(\pi) U(\pi, \underline{\gamma})
$$

and to calculate the ratio of normalization factors of the Gaussian measures $d \mu_{\gamma}^{0}(\Phi)$ and $d \mu^{0}(\Phi) \equiv d \mu_{y=\emptyset}^{0}(\Phi)$ (=standard free-field Gaussian measure with mass $\mathrm{m}^{2}$ ). This ratio of normalization factors is formally given by

$$
\operatorname{det}\left(-\Delta_{\gamma}+m^{2}\right)^{-1 / 2} / \operatorname{det}\left(-\Delta+m^{2}\right)^{-1 / 2}
$$

but this ratio vanishes. Introducing an ultraviolet regulator $\kappa$, e.g. a lattice cutoff, one may choose a function $\alpha(\kappa)$, diverging to $+\infty$, as $\kappa \rightarrow \infty$, such that

$$
z(\partial \underline{\gamma}) \equiv \lim _{\kappa \rightarrow \infty} \alpha(\kappa)^{2 n} \operatorname{det}\left(\left(-\Delta_{\underline{\gamma}}^{(\kappa)}+m^{2}\right) /\left(-\Delta^{(\kappa)}+m^{2}\right)\right)^{-1 / 2}
$$

exists, as long as $\min _{i \neq 1} \operatorname{dist}\left(x_{i}, x_{j}\right)>0$. The function $z(\partial \underline{\gamma})$ is gauge-invariant and therefore only depends on $\hat{\partial} \underline{\gamma}=\left\{x_{1}, \ldots, x_{2 n}\right\}$, but not on our choice of $\underline{\gamma}$. It can be calculated essentially explicitly, and one can show that

$$
z(\partial \gamma) \underset{d_{n} \rightarrow 0}{\sim} \exp \left(\operatorname{const}\left[\log d_{n}\right]^{2}\right),
$$

where $d_{n}=\min _{i \neq j} \operatorname{dist}\left(x_{i}, x_{j}\right)$. 


\section{Defining}

$$
V_{ \pm}(\Phi)=\frac{\lambda}{4}\left[\int d^{2} x:\left(\Phi^{2}-\xi\right)^{2}:(x)-\left(2 m^{2} / \lambda\right): \Phi^{2}:(x)\right]
$$

with + or - b.c. at infinity (see $[5 b, 9]$ ), we see that the formal expression $(2.36)$ is really given by

$$
G_{s, \pm}^{(2 n, m)}\left(x_{1}, \ldots, x_{2 n} ; y_{1}, \ldots, y_{m}\right)=\frac{\int d \mu_{\gamma}^{0}(\Phi) e^{-V_{ \pm}(\Phi)} \prod_{j=1}^{m} \Phi_{\gamma}\left(y_{j}\right)}{\int d \mu^{0}(\Phi) e^{-V_{ \pm}(\Phi)}} z(\partial \underline{\gamma}) .
$$

The first quotient on the right-hand side of (2.40) can be defined, mathematically precisely, by using fairly standard methods of constructive field theory [5a, 6]. It is finite as long as $y_{i} \neq y_{j}$, for $i \neq j$, with logarithmic singularities, as $\left|y_{i}-y_{j}\right| \rightarrow 0$. The details of these constructions are carried out in [9].

Our notation on the left-hand side of (2.36), (2.40) suggests that the right-hand side of $(2.40)$ only depends on $\partial \underline{\gamma}=\left\{x_{1}, \ldots, x_{2 n}\right\}$. This is indeed the case and follows from gauge-invariance.

It is instructive to compare the expressions (2.36) and (2.40) with the corresponding expressions for the lattice theory [2]. We set

$$
Z=\int \exp \left[-\frac{1}{2} \sum_{\langle i j\rangle}\left(\Phi_{i}-\Phi_{j}\right)^{2}\right] \prod_{j} d \varrho\left(\Phi_{j}\right)
$$

and

$$
G_{s, \pm}^{(2 n, m)}\left(x_{1}, \ldots, x_{2 n} ; y_{1}, \ldots, y_{m}\right)=\frac{\alpha^{2 n}}{Z} \int \exp \left[-\frac{1}{2} \sum_{\langle i j\rangle}\left(\Phi_{i}-\sigma_{i j} \Phi_{j}\right)^{2}\right] \prod_{k=1}^{m} \Phi_{y_{k}} \prod_{j} d \varrho\left(\Phi_{j}\right),
$$

where $\alpha(=\alpha(\kappa=1))$ is an arbitrary positive number, $x_{1}, \ldots, x_{2 n}$ belong to the dual lattice $\left(\mathbb{Z}^{2}\right)^{*}$, and $\sigma$ is a $\mathbb{Z}_{2}$ lattice gauge field given by

$$
\sigma_{i j}=\left\{\begin{aligned}
-1, & \text { if }\langle i j\rangle^{*} \in \gamma, \\
1, & \text { otherwise, }
\end{aligned}\right.
$$

where $\gamma=\left(\gamma_{1}, \ldots, \gamma_{n}\right)$ are $n$ paths in $\left(\mathbb{Z}^{2}\right)^{*}$ joining $n$ disjoint pairs of points in $\left\{x_{1}, \ldots, x_{2 n}\right\}$ (see [2]). By introducing a variable lattice spacing $a \equiv \kappa^{-1}$ and choosing $\alpha=\alpha(\kappa), d \varrho=d \varrho_{\kappa}$ (independent of $\gamma$ and $y_{1}, \ldots, y_{m}$ ) in a suitable way as functions of $\kappa$, one obtains (2.40) as the limit of (2.41), as $\kappa=a^{-1} \rightarrow \infty$. We have suppressed here the discussion of the thermodynamic limit; see (2.23). [Its existence is an easy consequence of FKG correlation inequalities [6], as the reader will check quite easily.]

The main result, proven in [9], is now that the Green functions $G_{s, \pm}^{(2 n, m)}\left(x_{1}, \ldots, x_{2 n} ; y_{1}, \ldots, y_{m}\right)$ satisfy the Osterwalder-Schrader axioms. This has been proven in detail for the lattice theory in [2]. The extension to the continuum limit is discussed in [9], and the main ideas are sketched below. It is worthwhile to note that, because of the singular behaviour found in (2.39), the Green functions $G_{s, \pm}^{(2 n, m)}\left(x_{1}, \ldots, x_{2 n} ; y_{1}, \ldots, y_{m}\right)$ are not tempered distributions, but belong to a Jaffe 
class of ultradistributions. An extension of Osterwalder-Schrader reconstruction to this class is known [10]; (see also [8]).

From the Osterwalder-Schrader reconstruction theorem it then follows that $\left\{G_{s, \pm}^{(2 n, m)}\right\}$ are the Euclidean Green functions of a local soliton field operator $s(x)$ and the meson field operator $\phi(x)$ : For $x_{1}^{0}<\ldots<x_{2 n}^{0}<y_{1}^{0}<\ldots<y_{m}^{0}$ we have

$$
\begin{aligned}
& G_{s, \pm}^{(2 n, m)}\left(x_{1}, \ldots, x_{2 n} ; y_{1}, \ldots, y_{m}\right)=\left\langle\Omega_{ \pm}, s\left(0, x_{1}^{1}\right) e^{-\left(x_{2}^{0}-x_{1}^{0}\right) H} \ldots s\left(0, x_{2 n}^{1}\right)\right. \\
& \left.\quad \times e^{-\left(y_{1}^{0}-x_{2 n}^{0}\right) H} \phi\left(0, y_{1}^{1}\right) e^{-\left(y_{2}^{0}-y_{1}^{0}\right) H} \phi\left(0, y_{2}^{1}\right) \ldots \phi\left(0, y_{m}^{1}\right) \Omega_{ \pm}\right\rangle .
\end{aligned}
$$

When $\xi>\xi_{c}$, so that

$$
\left\langle\Omega_{ \pm}, \phi(y) \Omega_{ \pm}\right\rangle= \pm \Phi_{c}, \quad \text { with } \quad \Phi_{c}>0,
$$

the physical Hilbert spaces reconstructed from (2.42) are

$$
\text { and } \left.\begin{array}{r}
\mathscr{H}_{\text {phys }}=\mathscr{H}_{+} \oplus \mathscr{H}_{s}, \\
\mathscr{H}_{\text {phys }}^{\prime}=\mathscr{H}_{-} \oplus \mathscr{H}_{\bar{s}} ;
\end{array}\right\} \text {. }
$$

$\mathscr{H}_{+}$and $\mathscr{H}_{-}$are the vacuum sectors which we have already discussed, while $\mathscr{H}_{s}$ and $\mathscr{H}_{\bar{s}}$ are a soliton- and an anti-soliton sector. [When $\xi$ is sufficiently negative, $\mathscr{H}_{+}$ $=\mathscr{H}_{-}=\mathscr{H}_{s}=\mathscr{H}_{\bar{s}}, \Omega_{+}=\Omega_{-} \equiv \Omega$, and $\langle\Omega, s(x) \Omega\rangle \neq 0$, while $\langle\Omega, \phi(y) \Omega\rangle=0$. This is the symmetric phase of the $\lambda \phi_{2}^{4}$-model. We shall not discuss it anymore, henceforth.]

The quantum field theories on $\mathscr{H}_{\text {phys }}$ and on $\mathscr{H}_{\text {phys }}^{\prime}$ are isomorphic, (identical particle spectra and identical dynamics), and are related to each other by the symmetry operation $\phi \rightarrow-\phi$. It therefore suffices to study the theory on $\mathscr{H}_{\text {phys }}$, and we shall omit the subscript + henceforth. For $\xi>\xi_{c}$, the Green functions $G_{s}^{(2 n, m)}\left(x_{1}, \ldots, x_{2 n} ; y_{1}, \ldots, y_{m}\right)$ have exponential cluster decomposition properties, with

$$
G_{s}^{(2 n, m)}\left(x_{1}, \ldots, x_{j}+a, \ldots, x_{2 n} ; y_{1}, \ldots, y_{m}\right) \rightarrow 0,
$$

as $|a| \rightarrow \infty$, exponentially fast [9]. Therefore, the soliton field has vanishing matrix elements between states in $\mathscr{H}_{0} \equiv \mathscr{H}_{+}$, or between states in $\mathscr{H}_{s}$, but non-zero matrix elements between states in $\mathscr{H}_{0}$ and states in $\mathscr{H}_{s}$. The spaces $\mathscr{H}_{0}$ and $\mathscr{H}_{s}$ are eigenspaces of the topological charge

$$
Q=\int_{-\infty}^{+\infty}\left(\frac{\partial}{\partial x^{1}} \phi\right)\left(x^{0}, x^{1}\right) d x^{1}
$$

with eigenvalues $0,2 \Phi_{c}$, respectively.

The mass gap (lowest eigenvalue $\neq 0$ of the mass operator $M$ ) on $\mathscr{H}_{0}$ is $m_{\text {phys }}$ $>0$, the lowest eigenvalue of $M$ on $\mathscr{H}_{s}$ is $m_{s}>0$, with $m_{\text {phys }} \propto \lambda \xi$ and $m_{s} \propto \sqrt{\xi}$, for $\xi \propto \frac{1}{\lambda}$ large enough; see [1c,9]. [The proofs of these results require a somewhat nontrivial extension of the methods in [5b].] It follows, in particular, that $\mathscr{H}_{s}$ does not contain any translation invariant states (vacua).

The meson- and soliton fields, $\phi$ and $s$, satisfy the "dual algebra," first discovered in [1a]: For $x$ and $y$ two space-like separated points in $\mathbb{M}^{2}$,

$$
\phi(x) s(y)=(-1)^{\theta\left(y^{1-x^{1}}\right)} s(y) \phi(x)
$$


where $\theta$ is the Heavyside step function. [Note that, in two space-time dimensions, $y^{1}-x^{1} \gtrless 0$ are Lorentz-invariant conditions if $x$ and $y$ are spacelike separated.] The dual algebra can be used to fermionize the $\lambda \phi_{2}^{4}$ model: We define a real, local field, $\psi(x)$, by

$$
\psi(x)=\phi(x) s(x)
$$

Then the algebra (2.44) implies

$$
\begin{aligned}
\psi(x) \psi(y) & =\phi(x) s(x) \phi(y) s(y)=(-1)^{\theta\left(x^{1}-y^{1}\right)} \phi(x) \phi(y) s(x) s(y) \\
& =(-1)^{\theta\left(x^{1}-y^{1}\right)} \phi(y) \phi(x) s(y) s(x) \\
& =(-1)^{\theta\left(x^{1}-y^{1}\right)}(-1)^{\theta\left(y^{1}-x^{1}\right)} \phi(y) s(y) \phi(x) s(x) \\
& =-\psi(y) \psi(x)
\end{aligned}
$$

for $x$ and $y$ space-like separated. [We have used that $\phi$ and $s$ are local Bose fields which follows from the symmetry of $G_{s}^{(2 n, m)}\left(x_{1}, \ldots, x_{2 n} ; y_{1}, \ldots, y_{m}\right)$ under exchanging $x$-, or $y$-arguments among themselves [7].] Relation (2.44') identifies $\psi$ as a Fermi field and, since it is real, $\psi$ describes a Majorana fermion. It couples the vacuum $\Omega$ to soliton states. Hence the soliton can be thought of as being a Majorana fermion. Another theory which can be described in terms of Majorana fermions is the twodimensional Ising model. One might think, therefore, that there must be a connection between $\lambda \phi_{2}^{4}$ and the two-dimensional Ising field theory, with $\phi(x)$ playing the role of the spin field in the Ising model, and $\psi(x)$ playing the role of Onsager's Majorana fermion in terms of which the Ising model is described as a free Majorana theory. Indeed, it is generally believed that the long-distance scaling limit of the Green functions of the $\lambda \phi_{2}^{4}$-model at the critical point $\left(\xi=\xi_{c}\right)$, i.e. the infrared fixed point of $\lambda \phi_{2}^{4}$, is identical to the critical Ising field theory.

The dual algebra (2.44) has the following consequences pointed out in [11]:

(1) $\langle\Omega, \phi(y) s(x) \Omega\rangle=0$, hence $\langle\Omega, \phi \Omega\rangle\langle\Omega, s \Omega\rangle=0$;

(2) $\langle\Omega, \phi \Omega\rangle=\langle\Omega, s \Omega\rangle=0$ implies $m_{\text {phys }}=0$, i.e. a critical theory.

These are model-independent results which follow from cluster properties of $\left\{G_{s}^{(2 n, m)}\right\}$ and (2.44).

We finally remark that the form factor

$$
\left\langle e^{-\varepsilon H} S\left(0, y^{1}\right) \Omega, \phi\left(0, x^{1}\right) e^{-\varepsilon H} S\left(0, \tilde{y}^{1}\right) \Omega\right\rangle
$$

resembles the classical kink solution of the $\lambda \phi_{2}^{4}$-model quoted in (2.6).

Most of these results have been established for the lattice theory in [2] and in the continuum limit in [9]; see also [1]. Here we just outline the main formal ideas of the proofs.

1. Gauge-invariance: $G_{s}^{(2 n, m)}$ depends on $\underline{\gamma}$ only through $\partial \underline{\gamma}=\left\{x_{1}, \ldots, x_{2 n}\right\}$. To see this, we define $\mathbb{Z}_{2}$-gauge transformations as follows. Partition $\mathbb{R}^{2}$ into two disjoint subsets, $B$ and $B^{c}$. We define $\varepsilon_{B}$ by

$$
\begin{aligned}
\left(\varepsilon_{B} \Phi\right)(x) & =\left\{\begin{array}{rr}
-\Phi(x), & x \in B \\
\Phi(x), & x \in B^{c},
\end{array}\right\} \\
\varepsilon_{B} \underline{\gamma} & =\underline{\gamma} \cup \partial B .
\end{aligned}
$$


Fig. 2

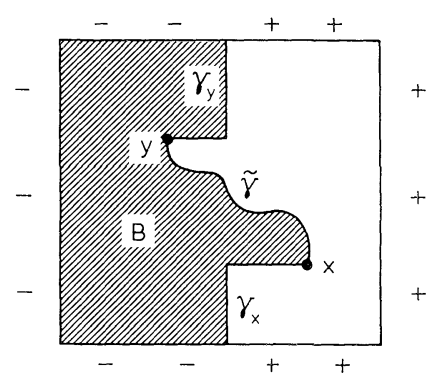

Then

$$
\begin{gathered}
\nabla_{\varepsilon_{B} \gamma}\left(\varepsilon_{B} \Phi\right)=\varepsilon_{B}\left(\nabla_{\gamma} \Phi\right), \\
\left(\varepsilon_{B} \Phi\right)^{2 p}=\Phi^{2 p}, \quad p=1,2, \ldots
\end{gathered}
$$

Hence

$$
S\left(\varepsilon_{B} \Phi, \varepsilon_{B} \underline{\gamma}\right)=S(\Phi, \underline{\gamma})
$$

Moreover,

$$
\Phi_{\underline{\gamma}}(x)=\left(\varepsilon_{B} \Phi_{\varepsilon_{B} \underline{\gamma}}\right)(x) \text { and } \quad D_{E} \Phi_{ \pm}=D_{E}\left(\varepsilon_{B} \Phi\right)_{ \pm},
$$

provided $B$ is compact.

Hence

$$
\left[\frac{1}{Z} \int_{\Gamma_{d}(E)} e^{-S(\Phi \cdot \hat{\gamma})} \prod_{j=1}^{m} \Phi_{\gamma}\left(y_{j}\right) D_{E} \Phi_{ \pm}\right]_{\mathrm{ren}}=\left[\frac{1}{Z} \int_{\Gamma_{d}(E)} e^{-S\left(\Phi^{\prime} \cdot \varepsilon_{B} \gamma\right)} \prod_{j=1}^{m} \Phi_{\varepsilon_{B \gamma} \gamma}^{\prime}\left(y_{j}\right) D_{E} \Phi_{ \pm}^{\prime}\right]_{\mathrm{ren}},
$$

which completes the proof.

Gauge invariance permits us to choose the paths $\gamma$ to reach out to infinity. Consider, for example, the soliton propagator $G_{s}^{(2)}(x, y)$, with $x^{0}<0<y^{0}$. We may then choose $\gamma=\gamma_{x} \cup \gamma_{y}$ to consist of a path $\gamma_{x}$ starting at $x$ and tending towards $(-\infty, 0)$ and of a path $\gamma_{y}$ starting at $y$ and tending towards $(+\infty, 0)$. In this case, we must, however impose mixed $(+-)$ b.c. at infinity, i.e. $D_{E} \Phi_{+}$is replaced by $D_{E} \Phi_{(+-)}$, as shown in Fig. 2.

By performing a "global" $\mathbb{Z}_{2}$ gauge transformation labelled by $B$, the new definition of $G_{s}^{(2)}(x, y)$ involving $(+-)$ b.c. at infinity, with $\gamma=\gamma_{x} \cup \gamma_{y}$, is seen to be equivalent to the definition of $G_{s}^{(2)}(x, y)$ given in (2.40) which involves pure + b.c. at infinity (or pure - b.c.), and with $\gamma=\tilde{\gamma}$. [These formal manipulations can be made precise by introducing a finite-volume cutoff, as shown in Fig. 2, and removing it at the end of the construction. See $[2,9]$.] The new definition is more akin to the Hamiltonian approach developed in [1].

2. Euclidean invariance and clustering: Formally, Euclidean invariance of the distributions $\left\{G_{s}^{(2 n, m)}\right\}$ is obvious. It can be proven by using FKG inequalities [6] or a field-theoretic version of the Peierls argument $[5,9]$ to construct the thermodynamic limit. The second method also proves exponential clustering. This is the main result of [9]. 
3. Osterwalder-Schrader positivity: For the soliton propagator, $G_{s}^{(2)}$, Osterwalder-Schrader positivity is the inequality

$$
\int d^{2} x d^{2} y G_{s}^{(2)}(x, y) \overline{f_{\theta}(x)} f(y) \geqq 0,
$$

for every test function $f$, with supp $f \cong\left\{\tilde{x}: \tilde{x}^{0}>0\right\}$, where $f_{\theta}\left(x^{0}, x^{1}\right) \equiv f\left(-x^{0}, x^{1}\right)$. If, in our construction of $G_{s}^{(2)}(x, y)$, we choose $\gamma=\gamma_{x} \cup \gamma_{y}$, as in Fig. 2, then formally

$$
G_{s}^{(2)}(x, y)=\int F_{\gamma_{x}}^{s g}(\Phi) F_{\gamma_{y}}^{s g}(\Phi) d \mu_{(+-)}(\Phi),
$$

where $F_{\gamma_{x}}^{s g}(\Phi)$ is real, singular functional of $\Phi$ concentrated along $\gamma_{x}$, with $F_{\gamma_{x}}^{s g}(\Phi) \in \mathscr{F}_{+}$, if $\gamma_{x} \subset\left\{\tilde{x}: \tilde{x}^{0}>0\right\}$. Now, for $x^{0}<0$,

$$
F_{\gamma x}^{s g}(\Phi)=\theta F_{\gamma \theta x}^{s g}(\Phi) \text {, with } \quad F_{\gamma_{\theta x}}^{s g}=F_{\theta\left(\gamma_{x}\right)}^{s g} \in \mathscr{F}_{+},
$$

where $\theta x=\left(-x^{0}, x^{1}\right)$, and $\theta\left(\gamma_{x}\right)$ is the time-reflection of $\gamma_{x}$. Therefore, inequality (2.47) follows from

$$
\int \overline{\theta F}(\Phi) F(\Phi) d \mu_{(+-)}(\Phi) \geqq 0,
$$

for all $F \in \mathscr{F}_{+}$, which is well known.

A more explicit proof of (2.47) has been given in [2] for the lattice $\lambda \phi_{2}^{4}$-model, and since the lattice approximation converges, (2.47) is true in the continuum limit. Osterwalder-Schrader positivity for the full set $\left\{G_{s}^{(2 n . m)}\right\}$ of Euclidean Green functions can be shown similarly, with more effort in keeping notations straight.

Next, we sketch the construction of the soliton sector $\mathscr{H}_{s} ;\left(\mathscr{H}_{\bar{s}}\right.$ is obtained in the same way by applying the symmetry operation $\phi \mapsto-\phi)$. We claim that the following states form a dense set in $\mathscr{H}_{s}$ :

$$
s(f)|F\rangle \equiv \int d^{2} x f(x) e^{-x^{0} H} s\left(0, x^{1}\right)|F\rangle, \quad F \in \mathscr{F}_{+},
$$

where $f$ is a test function with support in $\left\{\tilde{x}: \tilde{x}^{0}>0\right\}$. The scalar product of two states, $s(f)|F\rangle$ and $s(g)|G\rangle$, of the form (2.48) is given by

$$
\langle s(f) F|s(g)| G\rangle=\left[\iint d^{2} x d^{2} y \overline{f_{\theta}(x)} g(y)\left\langle(\overline{\theta F})_{-x^{0}} G_{y^{0}}\right\rangle_{\gamma,+}\right]_{\hat{\gamma} \gamma=\{\theta x, y\}},
$$

where

$$
\langle F\rangle_{\gamma,+}=\frac{\int d \mu_{\gamma}^{0}(\Phi) e^{-V+(\Phi)} F\left(\Phi_{\gamma}\right)}{\int d \mu^{0}(\Phi) e^{-V+(\Phi)}} \Omega(\partial \gamma) .
$$

Using the cluster properties of $\left\{G_{s,+}^{(2 n, m)}\right\}$, one shows quite easily that

$$
\langle s(f) F \mid G\rangle=0 \text {, i.e. } \mathscr{H}_{\mathrm{s}} \perp \mathscr{H}_{0},
$$

and

$$
s(f) s(g)|F\rangle \equiv \iint d^{2} x d^{2} y f(x) g(y) e^{-x^{0} H} s\left(0, x^{1}\right) e^{-y^{0} H} s\left(0, y^{1}\right)|F\rangle
$$

belongs to the vacuum sector $\mathscr{H}_{0}$ (see $[2,9]$ ).

4. The dual algebra (2.44): We consider the Green functions $G_{s}^{(2 n, m)}\left(x_{1}, \ldots, x_{2 n}\right.$; $\left.y_{1}, \ldots, y_{m}\right)$ defined in (2.35), (2.40), with $x_{2 n} \equiv x, y_{1} \equiv y$. It is convenient to choose $\gamma$ to consist of the union of $n$ paths $\underline{\gamma}^{\prime}$ and a path $\gamma_{x}$ starting at $x$ and tending to $(\infty, 0)$, as discussed in 1) and Fig. 2, above. We now compare the phases of $G_{s}^{(2 n, m)}\left(x_{1}, \ldots, x\right.$; $\left.y, \ldots, y_{m}\right)$, for two different choices of $x$ and $y$, with $x_{1}, \ldots, x_{2 n-1}$ and $y_{2}, \ldots, y_{m}$ fixed: 
(i) $x=x^{(0)}=\left(0, x^{1}\right), y=y^{(\varepsilon)}=\left(\varepsilon, y^{1}\right)$;

(ii) $x=x^{(\varepsilon)}=\left(\varepsilon, x^{1}\right), y=y^{(0)}=\left(0, y^{1}\right), \varepsilon>0$.

Then it follows from the definition of $\Phi_{\gamma}(y)$,

$$
\Phi_{\gamma}(y)=\Phi(y) U\left(\pi_{y}, \underline{\gamma}\right), \quad \text { see }(2.35),
$$

and from the definition, $(2.36),(2.40)$, of $G_{s}^{(2 n, m)}$ that

$$
\lim _{\varepsilon \triangleright 0} G_{s}^{(2 n, m)}\left(x_{1}, \ldots, x^{(0)} ; y^{(\varepsilon)}, \ldots, y_{m}\right)=\sigma \cdot \lim _{\varepsilon \downarrow 0} G_{s}^{(2 n, m)}\left(x_{1}, \ldots, x^{(\varepsilon)} ; y^{(0)}, \ldots, y^{m}\right),
$$

with

$$
\sigma=1, \quad \text { if } y^{1}>x^{1}, \quad \text { and } \sigma=-1 \text {, if } y^{1}<x^{1} \text {. }
$$

This is easy to see for our choice of $\underline{\gamma}$.

From (2.50) and the symmetry of $G_{s}^{(2 n, m)}\left(x_{1}, \ldots, x_{2 n} ; y_{1}, \ldots, y_{m}\right)$ under exchanging arguments the dual commutation relations $(2.45)$ follow by standard arguments of axiomatic field theory.

5. The topological charge $Q$ : Let $F \in \mathscr{F}_{+}$. We claim that $e^{-\varepsilon H} S\left(0, x^{1}\right)|F\rangle, \varepsilon>0$, is an eigenstate of the topological charge $Q$ defined in (2.27) with eigenvalue $2 \Phi_{c}$, while $|F\rangle$ is an eigenstate of $Q$ with eigenvalue 0 . [The last statement has been proven in (2.29).] As mentioned in 2) (Osterwalder-Schrader positivity), states of the form $e^{-\varepsilon H} S\left(0, x^{1}\right)|F\rangle, F \in \mathscr{F}_{+}$, generate a dense set of states in $\mathscr{H}_{s},[2,9]$. Thus, we must calculate

$$
\left\langle e^{-\varepsilon H} S\left(0, x^{1}\right) F\left|Q e^{-\varepsilon^{\prime} H} S\left(0, y^{1}\right)\right| G\right\rangle,
$$

with $F, G \in \mathscr{F}_{+}, \varepsilon, \varepsilon^{\prime}>0$.

Now, (2.51) can be expressed in terms of functional integrals: By definition (2.27) of $Q$ and (2.36), we have

$$
(2.51)=\lim _{w \rightarrow \infty}\left[\frac{1}{Z} \int_{\Gamma_{d}(E)} e^{-S(\Phi, \gamma)} \overline{(\theta F)_{-\varepsilon}(\Phi)} G_{\varepsilon^{\prime}}(\Phi)\left[\Phi_{\gamma}(0, w)-\Phi_{\gamma}(0,-w)\right] D_{E} \Phi_{+}\right] \text {ren },
$$

where $\gamma$ is a path joining $\left(\varepsilon^{\prime}, y^{1}\right)$ to $\left(-\varepsilon, x^{1}\right)$. By the definition of $\Phi_{\gamma}$, the right-hand side of (2.52) is given by

$$
\lim _{w \rightarrow \infty}\left[\frac{1}{Z} \int_{\Gamma_{d}(E)} e^{-S(\Phi, \gamma)} \overline{(\theta F)_{-\varepsilon}(\Phi)} G_{\varepsilon^{\prime}}(\Phi)[\Phi(0, w)+\Phi(0,-w)] D_{E} \Phi_{+}\right]_{\mathrm{ren}} .
$$

By the cluster properties of the measure $d \mu_{+}(\Phi)$ introduced in (2.9), and since supp $\gamma$ is compact, we see that

$$
(2.53)=\left[\frac{1}{Z} \int_{\Gamma_{d}(E)} e^{-S(\Phi, \gamma)} \overline{(\theta F)_{-\varepsilon}(\Phi)} G_{\varepsilon^{\prime}}(\Phi) D_{E} \Phi_{+}\right]_{\mathrm{ren}} 2 \int d \mu_{+}(\Phi) \Phi(0) .
$$

The first factor on the right-hand side of (2.54) is equal to

$$
\left\langle e^{-\varepsilon H} S\left(0, x^{1}\right) F\left|e^{-\varepsilon^{\prime} H} S\left(0, y^{1}\right)\right| G\right\rangle,
$$


by (2.36), (2.40), while the second factor is

$$
2\left\langle\Omega_{+}, \phi(0) \Omega_{+}\right\rangle=2 \Phi_{c} .
$$

Hence

$$
(2.51)=2 \Phi_{c}\left\langle e^{-\varepsilon H} S\left(0, x^{1}\right) F\left|e^{-\varepsilon^{\prime} H} S\left(0, y^{1}\right)\right| G\right\rangle,
$$

which completes our proof of the claim that $\mathscr{H}_{s}$ is an eigenspace of $Q$ with eigenvalue $2 \Phi_{c}$.

6) The form factor: Let $F$ and $G$ be ain $\mathscr{F}_{+}$. We consider the function

$$
g(w)=\left\langle e^{-\varepsilon H} S\left(0, x^{1}\right) F\left|\phi(0, w) e^{-\varepsilon^{\prime} H} S\left(0, y^{1}\right)\right| G\right\rangle,
$$

with $\varepsilon, \varepsilon^{\prime}>0$. By a calculation essentially identical to the one carried out in 5), above, one shows that

$$
\lim _{w \rightarrow \pm \infty} g(w)= \pm \Phi_{c} .
$$

Thus, the form factor $g(w)$ resembles the kink solution of the classical field equations of the $\lambda \phi_{2}^{4}$ model.

Remark. For the lattice $\lambda \phi_{2}^{4}$ model, we have shown in [2] that the soliton is a massive, stable particle by showing that the soliton propagators $G_{s}^{(2)}(x, y)$ has a Källen-Lehmann spectral representation of the form

$$
G_{s}^{(2)}(x, y)=\int_{\mathbb{R}_{+}} d \varrho(a)\left(-\Delta+a^{2}\right)^{-1}(x, y),
$$

where $d \varrho$ is a positive measure of the form

$$
\begin{gathered}
d \varrho(a)=\approx \delta\left(a-m_{s}\right) d a+d \varrho^{\prime}(a), \\
z>0, \quad \operatorname{supp} d \varrho^{\prime} \subseteq\left[m_{\mathrm{s}}+\mu_{s}, \infty\right),
\end{gathered}
$$

with $0<m_{s}$, and $0<\mu_{s}\left(\approx m_{\text {phys }}\right.$, for $\lambda$ small, and $\xi \approx \frac{1}{\lambda}$ large $)$. While the methods in [2] do not directly apply to continuum theories, it is likely that one could improve them to prove (2.56) and (2.57) for continuum $\lambda \phi_{2}^{4}$. See also [19] for a general new approach to particle structure analysis.

One might ask whether the particle structure (soliton mass, soliton-meson bound states ...) of the theory can be investigated, quantitatively, with the help of some asymptotic expansion methods, in particular the semi-classical method? This appears to be possible, indeed, and here is the scheme: As an example, we consider a two-soliton Green function

$$
G_{s}^{(2, m)}\left(x, y ; z_{1}, \ldots, z_{m}\right)=\left[\frac{1}{Z} \int_{\Gamma_{d}(E)} e^{-S(\Phi, \gamma)} \prod_{i=1}^{m} \Phi_{\gamma}\left(z_{i}\right) D_{E} \Phi_{+}\right]_{\mathrm{ren}},
$$

see (2.36), (2.40). Here $\gamma$ is a path connecting $x$ to $y$. In order to evaluate (2.58) within a semiclassical approximation, we must start by looking for the critical points of the classical co-variant action

$$
S_{c}(\Phi, \gamma)=\int d^{2} x\left\{\frac{1}{2}\left(\nabla_{\gamma} \Phi\right)^{2}(x)+\frac{\lambda}{4}\left(\Phi^{2}-\xi\right)^{2}(x)\right\}
$$


on a Sobolev space

$$
\left\{\Phi: \int d^{2} x\left(\nabla_{\gamma} \Phi\right)^{2}(x)<\infty, \Phi(x) \rightarrow \sqrt{\xi} \text {, as }|x| \rightarrow \infty\right\}
$$

of sections of the soliton bundle. This is a well-posed variational problem. For a general connection supported on $\gamma=\left\{\gamma_{1}, \ldots, \gamma_{n}\right\}, n \geqq 2$, this appears to be a rather difficult problem, but for $n=1, \underline{\gamma}=\gamma$, it is equivalent to the following simpler problem: Let $\gamma_{0}$ be the shortest path connecting $x$ to $y$. We define the function space

$$
\begin{aligned}
\mathscr{F}_{\gamma_{0}}= & \left\{\Phi: \int d^{2} x(\nabla \Phi)^{2}(x)<\infty, \Phi(x) \rightarrow \sqrt{\xi}, \text { as }|x| \rightarrow \infty,\right. \\
& \text { and } \left.\Phi(x) \rightarrow 0, \text { as } x \rightarrow \gamma_{0}\right\} .
\end{aligned}
$$

We look for a function $\Phi_{0} \in \mathscr{F}_{\gamma_{0}}$ such that

$$
S_{c}\left(\Phi_{0}\right)=\inf _{\Phi \in \mathscr{F}_{\gamma_{0}}} S_{c}(\Phi)
$$

where $S_{c}(\Phi)=\int d^{2} x\left\{\frac{1}{2}(\nabla \Phi)^{2}(x)+\frac{\lambda}{4}\left(\Phi^{2}-\xi\right)^{2}(x)\right\}$ is the ordinary classical action. The minimizer $\Phi_{0}$ is clearly a section of the soliton bundle $E$, and it turns out to minimize $S_{c}(\Phi, \gamma)$, for $\Phi \in \Gamma(E)$. Once we have constructed $\Phi_{0}$, we expand $S(\Phi)$ around $\Phi_{0}$, for $\Phi \in \Gamma_{d}(E)$, i.e.

$$
S(\Phi)=S\left(\Phi_{0}+\delta \Phi\right)=S_{c}\left(\Phi_{0}\right)+\frac{1}{2} Q_{\gamma_{0}}(\delta \Phi)+O\left(\delta \Phi^{3}\right),
$$

where $\delta \Phi \in \Gamma_{d}(E)$, and $Q_{\gamma_{0}}$ is a positive quadratic form. Since $x, y$ and $\gamma_{0}$ are fixed, $Q$ does not have any zero modes. Moreover, in the limit, where $\operatorname{dist}\left(\operatorname{supp} \delta \Phi, \gamma_{0}\right) \rightarrow \infty$,

$$
Q_{\gamma_{0}}(\delta \Phi) \rightarrow \int d^{2} x\left\{(\nabla \delta \Phi)^{2}(x)+m^{2}(\delta \Phi)^{2}(x)\right\}
$$

with $m^{2}=2 \lambda \xi$. To one-loop order, the problem now reduces to calculating the quotient

$$
\operatorname{det}\left(Q_{\gamma_{0}}\right)^{-1 / 2} / \operatorname{det}\left(-\Delta+m^{2}\right)^{-1 / 2},
$$

and discussing the Gaussian measure with mean 0 and covariance $Q_{\gamma_{0}}^{-1}$.

This concludes our discussion of soliton quantization in the $\lambda \phi_{2}^{4}$ model. We have presented rather many details, because this example, although simple, is really quite basic. See $[1,2,9]$ for more mathematical details.

It should be emphasized that the theory developed in this section has a fairly straightforward extension to $\lambda \phi^{2 p}$-type theories, with $p=2,3,4, \ldots$, where $\phi$ is a complex or an $N \geqq 3$ component scalar field, and the Lagrangian has a discrete (but not necessarily abelian) internal symmetry group. Examples are the four states Potts field theory, or theories with internal $\mathbb{Z}_{p}$ symmetry, $p=3,4,5, \ldots$. These are theories with soliton fields obeying "parastatistics." Their Euclidean Green functions of soliton and meson fields have, in general, a more complicated monodromy structure than those of the $\lambda \phi_{2}^{4}$ - or Ising field theories and define quite interesting representations of the (pure) braid groups (see e.g. [20, 21]). This will be discussed in more detail elsewhere. 


\section{Some Comments on Chiral Bosonization and Spin Fields}

In this section, we briefly review bosonization of massless, free Fermi fields in the Euclidean formulation of quantum field theory and comment on the construction of disorder fields and spin fields. We also indicate how the formalism can be extended to more general Riemann surfaces. We realize that a general understanding of these issues has recently been achieved in [22], but for our arguments in the fifth section (the main section of this paper) a more down-to-earth and explicit version of these matters is useful. We thus elaborate on the formulation of the problem in [15].

Let $\psi$ be a free, relativistic Dirac field on two-dimensional Minkowski space $\mathbb{M}^{2}$ and $\bar{\psi}=\psi^{*} \gamma_{0}$, where $\psi^{*}$ is the adjoint Dirac field. The Feynman propagator is given by

$$
\left\langle\Omega_{0}, T\left[\bar{\psi}_{\alpha}(x) \psi_{\beta}(y)\right] \Omega_{0}\right\rangle=(i \not \partial+m)_{\alpha \beta} D_{m}(x-y),
$$

where $D_{m}$ is the Feynman propagator of the free scalar field of mass $m, \not \partial=\gamma_{R}^{0} \partial_{0}$ $+\gamma_{R}^{1} \partial_{1}$, and $\gamma_{R}^{0}, \gamma_{R}^{1}$ are the two-dimensional $2 \times 2$ Dirac matrices. It is convenient to choose a Majorana representation:

$$
\gamma_{R}^{0}=-i \sigma_{2}, \quad \gamma_{R}^{1}=\sigma_{1},
$$

where $\sigma_{1}, \sigma_{2}, \sigma_{3}$ are the usual Pauli matrices. Hence $\gamma_{R}^{0}$ and $\gamma_{R}^{1}$ are real matrices. We set

$$
\gamma_{R}^{5}=\gamma_{R}^{0} \gamma_{R}^{1}=\sigma_{3}
$$

When $m=0$, the Dirac equation splits into equations for left- and right-moving modes:

We set

$$
\psi=\left(\begin{array}{c}
\psi_{L} \\
\psi_{R}
\end{array}\right), \quad \psi_{L}=\left(\frac{1+\gamma_{R}^{5}}{2}\right) \psi, \quad \psi_{R}=\left(\frac{1-\gamma_{R}^{5}}{2}\right) \psi,
$$

and the Dirac equation reads

$$
\left(\partial_{0}+\partial_{1}\right) \psi_{L}=0, \quad\left(-\partial_{0}+\partial_{1}\right) \psi_{R}=0 .
$$

Hence we have a Majorana-Weyl representation. By Wick's theorem, the entire field theory can be reconstructed from (3.1).

We now pass from the Minkowski to the Euclidean region by performing a Wick rotation, $t \rightarrow i t$. We set $t=x^{0}$, with $\hat{x}=\left(x^{0}, x^{1}\right) \in \mathbb{E}^{2}$, and, accordingly, replace $\gamma_{R}^{0}$ by $\gamma^{0}=-i \gamma_{R}^{0}=\sigma_{2}, \gamma_{R}^{1}$ by $\gamma^{1}=\gamma_{R}^{1}=\sigma_{1}$ and $\gamma_{R}^{5}$ by $\gamma^{5}=-i \gamma^{0} \gamma^{1}=\sigma_{3}$. The Euclidean Dirac operator is then given by

$$
\not{\phi}=\gamma^{0} \partial_{0}+\gamma^{1} \partial_{1}=\left(\begin{array}{cc}
0 & i \partial_{0}+\partial_{1} \\
-i \partial_{0}+\partial_{1} & 0
\end{array}\right)=2\left(\begin{array}{ll}
0 & \overline{\hat{C}} \\
\partial & 0
\end{array}\right),
$$

where we have set $x \equiv i x^{0}+x^{1}, \bar{x} \equiv-i x^{0}+x^{1}, \partial=\frac{\partial}{\partial x}, \bar{\partial}=\frac{\partial}{\partial \bar{x}}$.

The Euclidean two-point function is therefore given by

$$
\left\langle\bar{\psi}_{\alpha}(\hat{x}) \psi_{\beta}(\hat{y})\right\rangle=(i \not \partial+m)_{\alpha \beta} G_{m}(\hat{x}-\hat{y}),
$$


where $G_{m}$ is the Green function of $-\Delta+m^{2}$. When $m$ tends to $0,(3.6)$ yields

$$
\left.\begin{array}{l}
\left\langle\bar{\psi}_{1}(\hat{x}) \psi_{2}(\hat{y})\right\rangle=\frac{i}{2 \pi}(\bar{x}-\bar{y})^{-1} \\
\left\langle\bar{\psi}_{2}(\hat{x}) \psi_{1}(\hat{y})\right\rangle=\frac{i}{2 \pi}(x-y)^{-1}
\end{array}\right\}
$$

From now on we shall not distinguish between the point $\hat{x} \in \mathbb{E}^{2}$ and the complex number $x=i x^{0}+x^{1}$, anymore, and we use the notations

$$
\left.\begin{array}{l}
\psi_{1}=\left(\frac{1+\gamma^{5}}{2}\right) \psi \equiv \psi_{L} \equiv b \\
\psi_{2}=\left(\frac{1-\gamma^{5}}{2}\right) \psi \equiv \psi_{R} \equiv \bar{c} .
\end{array}\right\}
$$

Then (3.7) reads

and

$$
\left.\begin{array}{l}
\langle\bar{b}(x) \bar{c}(y)\rangle=\frac{i}{2 \pi}(\bar{x}-\bar{y})^{-1}, \\
\langle c(x) b(y)\rangle=\frac{i}{2 \pi}(x-y)^{-1}
\end{array}\right\}
$$

$$
\langle\bar{b} b\rangle=\langle\bar{c} c\rangle=\langle\bar{b} c\rangle=\langle b \bar{c}\rangle=0,
$$

which follows from $\left\langle\bar{\psi}_{\alpha} \psi_{\alpha}\right\rangle=\left\langle\psi_{\alpha} \psi_{\beta}\right\rangle=\left\langle\bar{\psi}_{\alpha} \bar{\psi}_{\beta}\right\rangle=0$.

By applying Wick's theorem and using the anti-commutation properties of $b, c$, $\bar{b}$ and $\bar{c}$, we obtain

$$
\left\langle\prod_{j=1}^{n} \bar{b}\left(x_{j}\right) \bar{c}\left(y_{j}\right)\right\rangle=\left(\frac{i}{2 \pi}\right)^{n} \operatorname{det}\left(\frac{1}{\bar{x}_{i}-\bar{y}_{j}}\right)
$$

and

$$
\left\langle\prod_{j=1}^{n} b\left(x_{j}\right) c\left(y_{j}\right)\right\rangle=\left(-\frac{i}{2 \pi}\right)^{n} \operatorname{det}\left(\frac{1}{x_{i}-y_{j}}\right) .
$$

Formally, all these Green functions can be derived from the action

$$
S(\bar{\psi}, \psi)=\int(\bar{\psi} \not \psi \psi)(x) d^{2} x=\int[(\bar{b} \bar{\partial} \bar{c})(x)+(c \partial b)(x)] d^{2} x
$$

by Berezin integration, (with $\left.\left(\partial^{-1}\right)(x, y) \equiv \frac{i}{2 \pi}(x-y)^{-1} \ldots\right)$.

The theory is invariant under gauge transformations of the first kind

$$
U(\alpha) \psi=e^{i \frac{\alpha}{2}} \psi, \quad \alpha \in[0,2 \pi],
$$

and, for $m=0(!)$, under chiral rotations

$$
V(\alpha) \psi=e^{i \frac{\alpha}{2} \gamma^{5}} \psi, \quad \alpha \in[0,2 \pi] .
$$


Under these transformations, the chiral fields, $b, c, \bar{b}$, and $\bar{c}$ transform as follows:

$$
\left.\begin{array}{ll}
U(\alpha) b=e^{i \frac{\alpha}{2}} b, & U(\alpha) c=e^{-i \frac{\alpha}{2} c}, \\
U(\alpha) \bar{b}=e^{-i \frac{\alpha}{2} \bar{b},} & U(\alpha) \bar{c}=e^{i \frac{\alpha}{2} \bar{c}}
\end{array}\right\}
$$

and

$$
\left.\begin{array}{ll}
V(\alpha) b=e^{i \frac{\alpha}{2}} b, & V(\alpha) c=e^{-i \frac{\alpha}{2}} c, \\
V(\alpha) \bar{b}=e^{i \frac{\alpha}{2}} \bar{b}, & V(\alpha) \bar{c}=e^{-i \frac{\alpha}{2} \bar{c}}
\end{array}\right\}
$$

The chiral densities are given by

$$
\bar{\psi}\left(\frac{1+\gamma^{5}}{2}\right) \psi=\bar{b} b, \quad \bar{\psi}\left(\frac{1-\gamma^{5}}{2}\right) \psi=c \bar{c} .
$$

[By (3.9), no normal ordering is necessary, since contractions between $\bar{b}$ and $b$, or $c$ and $\bar{c}$, vanish.] Finally, the vector current $j^{\mu}$ and the axial vector current $j_{5}^{\mu}$ are given by

$$
\begin{gathered}
j^{0}=N\left(\bar{\psi} \gamma^{0} \psi\right)=i N(\bar{b} \bar{c}-c b), \\
j^{1}=N\left(\bar{\psi} \gamma^{1} \psi\right)=N(\bar{b} \bar{c}+c b), \\
j_{5}^{0}=-i N\left(\bar{\psi} \gamma^{0} \gamma^{5} \psi\right)=-N(\bar{b} \bar{c}+c b), \\
j_{5}^{1}=-i N\left(\bar{\psi} \gamma^{1} \gamma^{5} \psi\right)=i N(\bar{b} \bar{c}-c b),
\end{gathered}
$$

where $N$ is the usual normal (or Wick) ordering. Hence, setting $A=i A^{0}+A^{1}$, $\bar{A}=-i A^{0}+A^{1}$, we have that

and

$$
\left.\begin{array}{l}
j=-N(b c)=i j_{5}, \\
\bar{j}=N(\bar{b} \vec{c})=-i \bar{j}_{5} .
\end{array}\right\}
$$

The following simple identities - which follow quite easily from (3.10), (3.11) have played an important role in guiding the way to bosonization.

(A) Using (3.14), (3.9) and then (3.10), (3.11), we get

$$
\begin{aligned}
& \left\langle\prod_{i=1}^{n}\left\{\left(\bar{\psi}\left(\frac{1+\gamma^{5}}{2}\right) \psi\right)\left(x_{i}\right) \cdot\left(\bar{\psi}\left(\frac{1-\gamma^{5}}{2}\right) \psi\right)\left(y_{i}\right)\right\}\right\rangle=\left\langle\prod_{i=1}^{n}\left\{(\bar{b} b)\left(x_{i}\right)(c \bar{c})\left(y_{i}\right)\right\}\right\rangle \\
& =\left\langle\prod_{i=1}^{n} \bar{b}\left(x_{i}\right) \bar{c}\left(y_{i}\right)\right\rangle\left\langle\prod_{i=1}^{n} b\left(x_{i}\right) c\left(y_{i}\right)\right\rangle=\left(\frac{1}{2 \pi}\right)^{2 n}\left|\operatorname{det}\left(\frac{1}{x_{i}-y_{i}}\right)\right|^{2}
\end{aligned}
$$

(B) Let $A \equiv\left(a_{i j}\right)$, with $a_{i j}=\left(x_{i}-x_{j}\right)^{-1}$, for $i \neq j, a_{\imath i}=0$, for all $i$. Then, since $\operatorname{det} A=\operatorname{det} A^{T}=\operatorname{det}(-A)=(-1)^{\operatorname{size} A} \operatorname{det} A, \operatorname{det} A$ vanishes, unless size $A=2 n$ is even, i.e. $A$ is a $2 n \times 2 n$ matrix, in which case

$$
\operatorname{det} A=\sum_{\sigma: \sigma(i) \neq i} \operatorname{sign} \sigma \prod_{j=1}^{2 n} a_{j \sigma(j)}=\sum_{\text {pairings, } p} \prod_{j=1}^{n} a_{p(2 j-1) p(2 j)}^{2} .
$$

Evaluating $\left\langle\prod_{i=1}^{n} N(b c)\left(x_{i}\right)\right\rangle$ by using Wick's theorem and these facts, we readily 
find

$$
\left\langle\prod_{i=1}^{2 n+1} N(b c)\left(x_{i}\right)\right\rangle=0
$$

and

$$
\left\langle\prod_{i=1}^{2 n} N(b c)\left(x_{i}\right)\right\rangle=\sum_{\text {pairings }, p}\left(\frac{1}{2 \pi}\right)^{2 n} \prod_{i=1}^{n}\left(\frac{1}{x_{p(2 i-1)}-x_{p(2 i)}}\right)^{2} .
$$

Similar identities hold if $N(b c)$ is replaced by $N(\bar{b} \bar{c})$. Moreover, by (3.9),

$$
\left\langle\prod_{i=1}^{k} N(b c)\left(x_{i}\right) \prod_{j=1}^{l} N(\bar{b} \bar{c})\left(y_{j}\right)\right\rangle=\left\langle\prod_{i=1}^{k} N(b c)\left(x_{i}\right)\right\rangle\left\langle\prod_{j=1}^{l} N(\bar{b} \bar{c})\left(y_{j}\right)\right\rangle,
$$

at non-coinciding arguments. At coinciding arguments, there is a contribution from the anomaly. For example,

$$
\langle N(b c)(x) N(\bar{b} \bar{c})(y)\rangle=\frac{1}{4 \pi} \delta(x-y) .
$$

It is not hard to extend (3.19)-(3.21) to find the expressions for the Euclidean Green functions of arbitrary products of $N(b c)$-and $N(\bar{b} \bar{c})$-currents. [This yields a proof of Schwinger's formula

$$
\left.\left\langle\exp \left(i j^{\mu} A_{\mu}\right)\right\rangle=\exp \left[\frac{1}{2}\left\langle d A, \Delta^{-1} d A\right\rangle\right] .\right]
$$

(C) One may also derive, somewhat less easily, from (3.10) and (3.11) that if $F$ is a monomial in $\bar{b} b$ and $c \bar{c}$ then

$$
\langle N(b c)(x) F(\bar{b} b, c \bar{c})\rangle=\frac{i}{4 \pi^{2}} \int \frac{d^{2} y}{x-y}\left\langle\frac{\delta F(\bar{b} b, c \bar{c})}{\delta(\bar{b} b)(y)}(\bar{b} b)(y)-\frac{\delta F(\bar{b} b, c \bar{c})}{\delta(c \bar{c})(y)}(c \bar{c})(y)\right\rangle,
$$

and similarly for $\langle N(\bar{b} \bar{c}) F(\bar{b} b, c \bar{c})\rangle$.

A proof of (3.22) will emerge from our discussion of bosonization.

The identities derived in (A)-(C) permit us to calculate the Euclidean Green functions corresponding to arbitrary products of currents, axial-vector currents and chiral densities. [Identities (B) are the most fundamental ones. They extend to general Riemann surfaces.]

Next, we review the bosonization of the $b-c$ system described above. This is a well known and much exploited procedure. Since it will turn out to be very useful for the material in Sects. 4 and 5, it is worthwhile to briefly present our views of these matters.

We define the zero-mass Gaussian measure, $d \mu^{0}$ with mean 0 and covariance " $4 \pi(-4)^{-1}$ " (see [12]) in terms of its characteristic functional:

$$
\left\langle e^{i\langle\chi, f\rangle}\right\rangle^{0} \equiv \int_{\mathscr{S}^{\prime}} d \mu^{0}(\chi) e^{i\langle\chi, f\rangle}=e^{2 \pi\left\langle f, \Delta^{-1} f\right\rangle},
$$

if $f$ is a test function whose Fourier transform, $\hat{f}$, vanishes at the origin, and

$$
\int_{\mathscr{S}^{\prime}} d \mu^{0}(\chi) e^{i\langle\chi, f\rangle}=0
$$


if $f$ is real and $\hat{f}(0) \neq 0$. [In (3.23), (3.24), the sample space $\mathscr{S}^{\prime}$ is chosen to be the space of real, tempered distributions on $\mathbb{R}^{2}$. We shall simplify our notations by writing simply $\int$, instead of $\left.\int_{\mathscr{S}^{\prime}} \cdot\right]$

Heuristically

$$
d \mu^{0}(\chi)=\frac{1}{Z^{0}} \exp \left[-\frac{1}{8 \pi}\langle\nabla \chi, \nabla \chi\rangle\right] D \chi_{0},
$$

where $D \chi$ is the formal Lebesgue measure and the subscript " 0 " indicates that $\chi$ has zero-Dirichlet b.c. [i.e., symbolically, $\chi(x) \rightarrow 0$, as $|x| \rightarrow \infty$ ].

We define the Wick-ordered exponential, :exp $i\langle\chi, f\rangle$ :, by

$$
: e^{i\langle\chi, f\rangle}:=e^{i\langle\chi, f\rangle} e^{2 \pi\left\langle f,\left(-\Delta+m_{0}^{2}\right)^{-1} f\right\rangle},
$$

for some $m_{0}>0$. Then a straightforward calculation shows that, with $: e^{i \varepsilon x}:(x)$ $\equiv: e^{i \varepsilon\left\langle\chi, \delta_{x}\right\rangle}$,

$\int d \mu^{0}(\chi) \prod_{j=1}^{n}: e^{i \varepsilon_{j} \chi}:\left(x_{j}\right)=\left\{\begin{array}{l}0, \text { if } \sum_{i=1}^{n} \varepsilon_{i} \neq 0 \\ \prod_{i=1}^{n} c\left(m_{0}\right)^{\varepsilon_{i}^{2}} \exp \left[-2 \sum_{1 \leqq i<j \leqq n} \varepsilon_{i} \varepsilon_{j} \ln \left(\frac{1}{\left|x_{i}-x_{j}\right|}\right)\right],\end{array}\right.$

if $\sum_{i=1}^{n} \varepsilon_{i}=0$. Here $c\left(m_{0}\right)$ is a finite, positive constant depending on $m_{0}>0$, and we can choose $m_{0}$ such that $c\left(m_{0}\right)=(2 \pi)^{-1}$. These matters are discussed in some detail in [12].

We define a normal product, $N$, of Wick-ordered exponentials by setting

$$
\begin{aligned}
N\left(: e^{i\langle\chi, f\rangle}:: e^{i\langle\chi, g\rangle}:\right) & =e^{4 \pi\left\langle f,\left(-\Delta+m_{0}^{2}\right)^{-1} g\right\rangle}: e^{i\langle\chi, f\rangle}:: e^{i\langle\chi, g\rangle}: \\
& =: e^{i\langle\chi, f+g\rangle}:,
\end{aligned}
$$

where the second equation follows from (3.26).

Next, we define the disorder field. We start by noting that the action

$$
S(\chi)=\frac{1}{8 \pi} \int(\nabla \chi)^{2}(x) d^{2} \chi
$$

used in (3.25) is invariant under global, real shifts of $\chi$, hence, in analogy with Sect. 2, we define a soliton bundle as follows: We choose $k$ points, $\underline{w}=\left(w_{1}, \ldots, w_{k}\right)$, in $\mathbb{R}^{2}$ and set

$$
M_{\underline{w}}=\mathbb{R}^{2} \backslash\{\underline{w}\} .
$$

A soliton bundle is an $\mathbb{R}$-bundle with non-trivial flat connection in $M_{w}$, with the additive group action of $\mathbb{R}$. Note that, since $\mathbb{R}$ is not a discrete group, as $\mathbb{Z}_{2}$ in Sect. 2, we also need to specify a connection. Such bundles can be characterized by representations of the fundamental group of $M_{\underline{w}}$ with values in $\mathbb{R}$, i.e. by the holonomy of the connection.

With each $w_{i}$ we associate a real flux number $\Phi_{w_{t}} \equiv \Phi_{i}$ such that

$$
\sum_{i=1}^{k} \Phi_{i}=0
$$


Let $\omega$ be a loop in $M_{\underline{w}}$ and set

$$
\alpha_{\Phi}(\omega)=\sum_{i=1}^{k} \Phi_{i} n\left(\omega, w_{i}\right)
$$

where $n\left(\omega, w_{i}\right)$ is the oriented winding number of $\omega$ with respect to $w_{i}$ (counterclockwise windings are counted positively, clockwise windings negatively). Then we denote by $E_{\Phi}$ the soliton bundle whose connection has holonomy $\alpha_{\Phi}$ given by (3.31). (Remember that, since $\mathbb{R}$ acts additively, the holonomy $\alpha_{\Phi}$ has an additive action, as well.) The covariant derivative on $E_{\Phi}$ is denoted by $\nabla_{\Phi}$. A concrete representation of the sections of $E_{\Phi}$ is obtained by considering the universal cover $\tilde{M}_{\underline{w}}$ of $M_{\underline{w}}$. Denote by $\pi$ the canonical projection: $\tilde{M}_{\underline{w}} \rightarrow M_{\underline{w}}$.

A section of $E_{\Phi}$ may be identified with a function $\tilde{\chi}$ on $\tilde{M}_{\underline{w}}$ such that if $\tilde{\gamma}$ is a path in $\tilde{M}_{\underline{w}}$ projecting to the loop $\omega$ in $M_{\underline{w}}$, i.e. $\pi(\tilde{\gamma})=\omega$, then

$$
\tilde{\chi}\left(\partial_{+} \tilde{\gamma}\right)=\tilde{\chi}\left(\partial_{-} \tilde{\gamma}\right)+\alpha_{\Phi}(\omega)
$$

In this representation the covariant derivative $\nabla_{\Phi}$ may be identified with the ordinary derivative on $\tilde{M}_{\underline{w}}$, and $\nabla_{\Phi} \tilde{\chi}$ globally projects down to a closed 1 -form $X$ on $M_{\underline{w}}$, i.e. $\nabla_{\underline{\Phi}} \tilde{\chi}=\pi^{*} X$, with $\nabla X=0$ on $M_{\underline{w}}$.

From now on we identify globally projectable forms with their projection, so that we write $\nabla_{\Phi} \tilde{\chi}=X$.

Since the space of sections of $E_{\Phi}$ is an affine space modelled on the space of real functions, every section $\tilde{\chi}$ can be written as the sum of a fixed section, $\tilde{\chi}_{0} \equiv \tilde{\alpha}$, and a function $\chi$. Hence, setting $\nabla_{\Phi} \tilde{\alpha} \equiv A_{\Phi}$, we have that

$$
\nabla_{\Phi} \tilde{\chi}=A_{\Phi}+\nabla \chi \text {. }
$$

The closed 1-form, $A_{\Phi}$ is a flat connection on $E_{\Phi}$. If we extend the 1-form $A_{\Phi}$ on $M_{\underline{w}}$ to a 1 -current (i.e. a 1 -form with coefficients in the distributions) on $\mathbb{R}^{2}$, then its curvature, $F_{\Phi}$ is concentrated in the points $\underline{w}$. In fact,

$$
F_{\Phi} \equiv \nabla A_{\Phi}=\sum_{i=1}^{k} \Phi_{i} \delta^{(2)}\left(x-w_{i}\right) d x^{0} \wedge d x^{1}
$$

From now on we generally omit the subscript $\Phi$ to simplify our notation.

There is a preferred choice for $A$ : We define

$$
\tilde{\alpha}(\tilde{x})=\tilde{\alpha}_{h}(\tilde{x})=\sum_{i=1}^{k} \frac{\Phi_{i}}{2 \pi} \phi\left(\tilde{x}, w_{i}\right)
$$

where $\phi\left(\tilde{x}, w_{i}\right)$ is the angle between the ray emanating from $w_{i}$ and passing through $\tilde{x}$ and the ray emanating from $w_{i}$ parallel to the positive $x^{1}$-half axis.

Notice that $A_{h}=\nabla \tilde{\alpha}_{h}$ can also be written as

$$
\begin{aligned}
A_{h}(x) & =\sum_{i=1}^{k} \Phi_{i}\left(* \nabla^{*} \Delta^{-1} \delta_{w_{l}}\right)(x) \\
& \equiv \sum_{i=1}^{k} \Phi_{i} \sum_{\mu, v} \varepsilon_{\mu}^{v}\left(\int \frac{\partial}{\partial x^{v}} \Delta^{-1}(x, y) \delta^{(2)}\left(w_{i}-y\right) d^{2} y\right) d x^{\mu} .
\end{aligned}
$$


This is a 1 -current in $\mathbb{R}^{2}$, but a smooth 1 -form in $M_{\underline{w}}$. Using (3.35), one immediately verifies that $A_{h}$ is harmonic on $M_{\underline{w}}$, i.e.

$$
\nabla A_{h}=* \nabla^{*} A_{h}=0 \text { on } M_{\underline{w}} .
$$

For later purposes it is worthwhile to note that if $j$ is a 1-current satisfying

$$
\nabla j=\sum_{i} \Phi_{i} \delta_{w_{\imath}} d x^{0} \wedge d x^{1}
$$

then, by the Hodge-de Rham decomposition

$$
j=\nabla \Delta^{-1 *} \nabla^{*} j+* \nabla^{*} \Delta^{-1} \nabla j=\nabla\left(\Delta^{-1 *} \nabla^{*} j\right)+A_{h},
$$

in the space of 1-currents.

We remark that, due to Eq. (3.31), every connection $A$ obtained by projecting some $\nabla_{\Phi} \tilde{x}$ satisfies the equation

$$
A-A_{h}=\nabla \alpha_{0},
$$

for some globally defined function $\alpha_{0}$.

To construct the disorder field, we now modify the action (3.29) as follows:

$$
S(\tilde{\chi})=\frac{1}{8 \pi} \int_{M_{w}}\left(\nabla_{\Phi} \tilde{\chi}\right)^{2} d^{2} x=S(\chi, A)=\frac{1}{8 \pi} \int_{M_{w}}(\nabla \chi+A)^{2} d^{2} x .
$$

We then define formally

$$
d \mu_{\tilde{\alpha}}^{0}(\chi) \equiv d \mu_{A}^{0}(\chi)=\left[\frac{1}{Z^{0}} e^{-S(\chi, A)}\right]_{\mathrm{ren}} D \chi_{0} .
$$

As in the $\lambda \phi_{2}^{4}$-model, the renormalization on the right-hand side of (3.40) is multiplicative.

In order to make mathematical sense of (3.40), we now define the Gaussian measure $d \mu_{A}^{0}$, via its generating functional: Let $f$ be a test function on $\mathbb{R}^{2}$, whose Fourier transform, $\hat{f}$, vanishes at $k=0$. Then, formally,

$$
\begin{aligned}
\int d \mu_{A}^{0}(\chi) e^{i\langle\chi, f\rangle} & =\int d \mu^{0}(\chi) e^{-\frac{1}{4 \pi}\langle\nabla \chi, A\rangle-\frac{1}{8 \pi}\langle A \cdot A\rangle} e^{i\langle\chi, f\rangle} \\
& =e^{-\frac{1}{8 \pi}\langle A, A\rangle} \exp \left[-2 \pi\left\langle\left(i f+\frac{{ }^{*} \nabla^{*} A}{4 \pi}\right), \Delta^{-1}\left(i f+\frac{{ }^{*} \nabla^{*} A}{4 \pi}\right)\right\rangle\right],
\end{aligned}
$$

where

$$
\langle f, g\rangle=\int_{M_{\underline{w}}} d^{2} x \overline{f(x)} g(x) .
$$

The problem with definition (3.41) is that $\langle A, A\rangle$ is logarithmically divergent, so that the right-hand side of (3.41) vanishes. To see this, we choose $A=A_{h}$, as in (3.34). Then

$$
\left\langle A_{h}, A_{h}\right\rangle=\frac{1}{2 \pi} \sum_{i, j=1}^{n} \Phi_{i} \Phi_{j} \ln \frac{1}{\left|w_{i}-w_{j}\right|} .
$$


Using Green's theorem and the fact that $A_{h}$ is harmonic, it is easy to see that

$$
\left\langle\nabla \chi, A_{h}\right\rangle=0 \text {, }
$$

provided $\chi$ is a single-valued function on $M_{\underline{w}}$. By (3.42) we may then define

$$
\begin{aligned}
\int d \mu_{A_{h}}^{0}(\chi) e^{i\langle\chi, f\rangle} & =e^{-\frac{1}{8 \pi}\left\langle A_{h}, A_{h}\right\rangle_{\mathrm{reg}}} e^{2 \pi\left\langle f, \Delta^{-1} f\right\rangle} \\
& =e^{-\frac{1}{8 \pi}\left\langle A_{h}, A_{h}\right\rangle \mathrm{reg}} \int d \mu^{0}(\chi) e^{i\langle\chi, f\rangle},
\end{aligned}
$$

where $d \mu^{0}$ is the zero-mass Gaussian defined in (3.32), and

$$
\left\langle A_{h}, A_{h}\right\rangle_{\mathrm{reg}}=\frac{1}{2 \pi} \sum_{i \neq j} \Phi_{i} \Phi_{j} \ln \frac{1}{\left|w_{i}-w_{j}\right|} \equiv \frac{1}{2 \pi} U(\underline{\Phi}, \underline{w}) .
$$

The definition of $d \mu_{A}^{0}$ for more general $A$ follows easily from (3.41), (3.43), (3.38) by setting

$$
\langle A, A\rangle_{\mathrm{reg}} \equiv\left\langle A_{h}, A_{h}\right\rangle_{\mathrm{reg}}+\left\langle\nabla \alpha_{0}, \nabla \alpha_{0}\right\rangle
$$

(the cross term $2\left\langle A_{h}, \nabla \alpha_{0}\right\rangle$ vanishes, since $A_{h}$ is harmonic), and we obtain

$$
\int d \mu_{A}^{0}(\chi) e^{i\langle\chi, f\rangle}=e^{-\frac{1}{8 \pi}\left\langle A_{h}, A_{h}\right\rangle_{\mathrm{reg}}} \exp \left[2 \pi\left\langle f, \Delta^{-1} f\right\rangle-i\left\langle f, \alpha_{0}\right\rangle\right]
$$

Let $f$ be a test function on $M_{w}$ whose support is contractible. On supp $f$ we may choose a branch $\bar{\alpha}$ of $\tilde{\alpha}$, i.e. $\overline{\bar{\alpha}}(x)$ is a single-valued function on supp $f$, with $\nabla \bar{\alpha}=A$. By (3.46)

$$
\int d \mu_{A}^{0}(\chi) e^{i\langle\chi+\bar{\alpha}, f\rangle}=\exp \left[-\frac{1}{16 \pi^{2}} U(\underline{\Phi}, \underline{w})\right] \exp \left[2 \pi\left\langle f, \Delta^{-1} f\right\rangle+i\left\langle f, \bar{\alpha}_{h}\right\rangle\right] .
$$

This result can easily be verified, heuristically, using (3.40). The right-hand side of (3.47) is manifestly gauge-invariant, (i.e. independent of the specific choice of $A$, subject to the conditions(3.38)). From (3.47) and (3.26) we obtain, for $\{\underline{w}\} \cap\left\{\underline{w}^{\prime}\right\}=\emptyset$,

$$
\int d \mu_{A}^{0}(\chi) \prod_{i=1}^{l}: e^{i \varepsilon_{i}(\chi+\bar{\alpha})}:\left(w_{i}^{\prime}\right)=G_{0}^{(k, l)}\left(\underline{\mathscr{B}} ; \underline{\Phi}, \underline{w} ; \underline{\varepsilon}, \underline{w}^{\prime}\right),
$$

where

$$
\begin{aligned}
G_{0}^{(k, l)}\left(\mathscr{B} ; \underline{\Phi}, \underline{w} ; \underline{\varepsilon}, \underline{w}^{\prime}\right) \equiv & \exp \left[-\frac{1}{16 \pi^{2}} U(\underline{\Phi}, \underline{w})\right] \\
& \times \exp \left[-\sum_{i \neq j} \varepsilon_{i} \varepsilon_{j} \ln \left(\frac{1}{\left|w_{i}^{\prime}-w_{j}^{\prime}\right|}\right)\right] \\
& \times \exp \left[i \sum_{i=1}^{l} \varepsilon_{i} \sum_{j=1}^{k} \frac{\Phi_{j}}{2 \pi} \phi_{\mathscr{B}_{J}}\left(w_{i}^{\prime}, w_{j}\right)\right],
\end{aligned}
$$

and $\phi_{\mathscr{B}_{J}}\left(\cdot, w_{j}\right)$ is a branch of $\phi\left(\cdot, w_{j}\right)$, indexed by $\mathscr{B}_{j}, j=1, \ldots, k$, and $\mathscr{\mathscr { B }} \equiv\left(\mathscr{B}_{j}\right)_{j=1}^{k}$. 
Next, we define disorder operators, $D(\mathscr{B}, \Phi, w)$, and an expectation $\langle(\cdot)\rangle^{0}$ by setting

$$
\begin{aligned}
& \left\langle\prod_{i=1}^{k} D\left(\mathscr{B}_{i}, \Phi_{i}, w_{i}\right) e^{i\langle\chi, f\rangle}\right\rangle^{0} \\
& \quad=\left\{\begin{array}{l}
0, \text { if } \sum_{i=1}^{k} \Phi_{i} \neq 0, \quad \text { or } \hat{f}(0) \neq 0, \quad f \text { real } \\
\exp \left[-\frac{1}{16 \pi^{2}} U(\underline{\Phi}, \underline{w})\right] \exp \left[2 \pi\left\langle f, \Delta^{-1} f\right\rangle+i\left\langle f, \bar{\alpha}_{h}\right\rangle\right], \quad \text { otherwise } .
\end{array}\right.
\end{aligned}
$$

Here

$$
\bar{\alpha}_{h}(x)=\sum_{j=1}^{k} \frac{\Phi_{j}}{2 \pi} \phi_{\mathscr{B}_{j}}\left(x, w_{j}\right) .
$$

If we agree to always write disorder operators to the left of functionals of $\chi$ we can define them, formally by setting

$$
D(\mathscr{B}, \Phi, w) F(\chi)=F(\chi+\bar{\alpha}) \frac{d \mu_{A}^{0}(\chi)}{d \mu^{0}(\chi)},
$$

with $\bar{\alpha}(\cdot)=\frac{\Phi}{2 \pi} \phi_{\mathscr{B}}(\cdot, w)+\alpha_{0}(\cdot)$, and $\frac{d \mu_{A}^{0}}{d \mu^{0}}$ is the formal Radon-Nikodym derivative of $d \mu_{A}^{0}$ with respect to $d \mu^{0}$.

We define a normal ordered product, *, by

$$
\begin{gathered}
D * F=F * D=D F \\
D * F * D^{\prime} * F^{\prime}=F * F^{\prime} * D * D^{\prime}=\ldots=D D^{\prime} F F^{\prime} .
\end{gathered}
$$

These notions and definitions permit us to develop a formal algebraic calculus with disorder operators.

Our purpose is now to use the formalism developed above to express the fermionic Green functions (3.10), (3.11), (3.16), (3.19), (3.22) in terms of the Gaussian field $\chi$ and the algebra of disorder operators, $D(\mathscr{B}, \Phi, w)$. We begin with the $n$-point functions of fermionic currents, (3.18)-(3.21).

Let $x=i x^{0}+x^{1}, \bar{x}=-i x^{0}+x^{1}, \partial=\frac{\partial}{\partial x}, \bar{\partial}=\frac{\partial}{\partial \bar{x}}, \partial^{\#}=\partial$, or $\bar{\partial}$. From formula (3.23) for the characteristic functional of the zero-mass Gaussian measure, $d \mu^{0}(\chi)$, it follows immediately that

$$
\left\langle\prod_{i=1}^{2 n+1}\left(\partial^{\#} \chi\right)\left(x_{i}\right)\right\rangle^{0}=0, \text { for all } n,
$$

and using that

$$
\left.\begin{array}{l}
\partial_{x} \partial_{y} 2 \ln \frac{1}{|x-y|}=\frac{1}{(x-y)^{2}}, \\
\bar{\partial}_{x} \bar{\partial}_{y} 2 \ln \frac{1}{|x-y|}=\frac{1}{(\bar{x}-\bar{y})^{2}}, \\
\partial_{x} \bar{\partial}_{x} 2 \ln \frac{1}{|x-y|}=\frac{1}{2} \Delta \ln \frac{1}{|x-y|}=-\pi \delta(x-y)
\end{array}\right\}
$$


we obtain from (3.23) that

$$
\begin{gathered}
\left\langle\prod_{i=1}^{2 n} \partial \chi\left(x_{i}\right)\right\rangle^{0}=\sum_{\text {pairings, } p} \prod_{i=1}^{n}\left(\frac{1}{x_{p(2 i-1)}-x_{p(2 i)}}\right)^{2}, \\
\left\langle\prod_{i=1}^{2 n} \bar{\partial} \chi\left(x_{i}\right)\right\rangle^{0}=\sum_{\text {pairings }, p} \prod_{i=1}^{n}\left(\frac{1}{\bar{x}_{p(2 i-1)}-\bar{x}_{p(2 i)}}\right)^{2}, \\
\langle\partial \chi(x) \bar{\partial} \chi(y)\rangle^{0}=\pi \delta(x-y), \quad \text { etc. }
\end{gathered}
$$

Comparison with (3.19)-(3.21) shows that we may identify

and

$$
\left.\begin{array}{lll}
N(b c) \text { with } & \frac{1}{2 \pi} \partial \chi \\
N(\bar{b} \bar{c}) \text { with } & \frac{1}{2 \pi} \bar{\partial} \chi .
\end{array}\right\}
$$

Thanks to (3.17), this identification extends to arbitrary Riemann surfaces; (we just must use the correct Green function of $-\Delta$ and its $\partial$ - and $\bar{\partial}$-derivatives). is

Next, we identify (3.27) with a fermionic Green function. A special case of (3.27)

$$
\begin{aligned}
\left\langle\prod_{j=1}^{n}: e^{i \chi}:\left(x_{j}\right): e^{-i \chi}:\left(y_{j}\right)\right\rangle^{0} \\
=\left(\frac{1}{2 \pi}\right)^{2 n} \exp \left\{-2 \sum_{1 \leqq i<j \leqq n}\left[\ln \left(\frac{1}{\left|x_{i}-x_{j}\right|}\right)\right.\right. \\
\left.\left.\quad+\ln \left(\frac{1}{\left|y_{i}-y_{j}\right|}\right)\right]+2 \sum_{i, j=1}^{n} \ln \left(\frac{1}{\left|x_{i}-y_{j}\right|}\right)\right\} \\
=\left(\frac{1}{2 \pi}\right)^{\frac{1 n}{1 \leqq i<j \leqq n}}\left|x_{i}-x_{j}\right|^{2}\left|y_{i}-y_{j}\right|^{2} \\
\prod_{i, j=1}^{n}\left|x_{i}-y_{j}\right|^{2}
\end{aligned}
$$

It is a well-known identity, due to Cauchy, that

$$
\frac{\prod_{i \leqq i<j \leqq n}\left(x_{i}-x_{j}\right)\left(y_{j}-y_{i}\right)}{\prod_{i, j=1}^{n}\left(x_{i}-y_{j}\right)}=\operatorname{det}\left(\frac{1}{x_{i}-y_{j}}\right) .
$$

From (3.58) and (3.59) we conclude that

$$
\left\langle\prod_{j=1}^{n}: e^{i \chi}:\left(x_{j}\right): e^{-i \chi}:\left(y_{i}\right)\right\rangle^{0}=\left(\frac{1}{2 \pi}\right)^{2 n}\left|\operatorname{det}\left(\frac{1}{x_{i}-y_{j}}\right)\right|^{2} .
$$

Comparison with (3.16) permits us to identify

and

$$
\left.\begin{array}{l}
{\left[\bar{\psi}\left(\frac{1+\gamma_{5}}{2}\right) \psi\right](x)=(\bar{b} b)(x) \text { with }: e^{i \chi}:(x)} \\
{\left[\bar{\psi}\left(\frac{1-y_{5}}{2}\right) \psi\right](x)=(c \bar{c})(x) \text { with }: e^{-i \chi}:(x) .}
\end{array}\right\}
$$


These identities were already observed in $[15]$; (see also $[12,14])$.

It is natural to ask whether the chiral Fermi fields $b, c, \bar{b}$ and $\bar{c}$ can be expressed in terms of the zero mass Bose field $\chi$, too? It turns out that they can be expressed in terms of $\chi$ and the disorder operators, $D(\mathscr{B}, \Phi, w)$.

To perform our calculations we choose $\tilde{\alpha}=\tilde{\alpha}_{h}$ as in (3.34), with

$$
\left.\begin{array}{rl}
\underline{w}=(\underline{x}, \underline{y}), \quad \underline{x}=\left(x_{1}, \ldots, x_{n}\right), & \underline{y}=\left(y_{1}, \ldots, y_{n}\right), \\
\Phi_{x_{i}}=2 \pi, \quad \Phi_{y_{1}}=-2 \pi, & i=1, \ldots, n .
\end{array}\right\}
$$

We now note that, on given branches of the functions $\phi(\cdot, x)$ and $\phi(\cdot, y)$, we have that

$$
\phi(x, y)=\phi(y, x)+(2 m+1) \pi, \quad m \in \mathbb{Z} .
$$

We define a branch, $\mathscr{B}_{0}$, of $\phi(\cdot, x)$ by setting $\phi(w, x)$ equal to the angle between the half line through $w$ and $x$ which starts at $x$ and the half line through $x$ parallel to the positive 1 -axis. Then

$$
\left.\begin{array}{lll}
\phi(x, y)=\phi(y, x)+\pi & \text { if } \quad y^{0}>x^{0} \\
\phi(x, y)=\phi(y, x)-\pi & \text { if } \quad y^{0}<x^{0}
\end{array}\right\}
$$

Suppose now that

$$
x_{1}^{0}<\ldots<x_{n-1}^{0}<x_{n}^{0}<y_{n}^{0}<y_{n-1}^{0}<\ldots<y_{1}^{0} .
$$

By formulas (3.48) and (3.50),

$$
\begin{aligned}
\lim _{\varepsilon \searrow 0}(2 \pi)^{n / 2} & \left\langle\prod_{i=1}^{n} *\left[: e^{i \frac{\chi}{2}}:\left(x_{i}+\varepsilon\right) D\left(\mathscr{B}_{0}, 2 \pi, x_{i}\right): e^{-i \frac{\chi}{2}}:\left(y_{i}+\varepsilon\right) D\left(\mathscr{B}_{0},-2 \pi, y_{i}\right)\right]\right\rangle^{0} \\
= & \exp \left[-\frac{1}{16 \pi^{2}} U(\underline{\Phi},(\underline{x}, \underline{y}))\right] \exp \left\{-\frac{1}{2} \sum_{1 \leqq i<j \leqq n}\left[\ln \frac{1}{\left|x_{i}-x_{j}\right|}+\ln \frac{1}{\left|y_{i}-y_{j}\right|}\right]\right. \\
& \left.+\frac{1}{2} \sum_{i, j=1}^{n} \ln \frac{1}{\left|x_{i}-y_{j}\right|}\right\} \exp i\left\{\sum_{1 \leqq i<j \leqq n}\left[\left(\phi\left(x_{i}, x_{j}\right)+\frac{\pi}{2}\right)+\left(\phi\left(y_{j}, y_{i}\right)+\frac{\pi}{2}\right)\right]\right. \\
& \left.-\sum_{i, j=1}^{n}\left(\phi\left(x_{i}, y_{j}\right)+\frac{\pi}{2}\right)\right\} .
\end{aligned}
$$

Since we have set $\Phi_{x_{i}}=2 \pi=-\Phi_{y_{i}}, i=1, \ldots, n$,

$$
U(\Phi,(\underline{x}, \underline{y}))=8 \pi^{2}\left(\sum_{1 \leqq i<j \leqq n}\left[\ln \frac{1}{\left|x_{i}-y_{j}\right|}+\ln \frac{1}{\left|y_{i}-y_{j}\right|}\right]-\sum_{i, j=1}^{n} \ln \frac{1}{\left|x_{i}-y_{j}\right|}\right),
$$

by definition (3.44) of $U(\Phi, \underline{w})$. Hence the first and the second factor on the righthand side of (3.65) are equal, and their product is given by

$$
\frac{\prod_{1 \leqq i<j \leqq n}\left|x_{i}-x_{j}\right|\left|y_{j}-y_{i}\right|}{\prod_{i, j=1}^{n}\left|x_{i}-y_{j}\right|} .
$$


Clearly the product of (3.67) and the third exponential on the right-hand side of (3.65) is equal to

$$
e^{-i n \frac{\pi}{2} \frac{\prod_{1 \leqq i<j \leqq n}\left(x_{i}-x_{j}\right)\left(y_{j}-y_{i}\right)}{\prod_{i, j=1}^{n}\left(x_{i}-y_{j}\right)}}=(-i)^{n} \operatorname{det}\left(\frac{1}{x_{i}-y_{j}}\right),
$$

by Cauchy's identity (3.59).

Comparison with (3.11) finally proves that

$$
\begin{gathered}
\lim _{\varepsilon \triangleright 0}(2 \pi)^{-n / 2}\left\langle\prod_{i=1}^{2 n} *\left[: e^{i \frac{\chi}{2}}:\left(x_{i}+\varepsilon\right) D\left(\mathscr{B}_{0}, 2 \pi, x_{i}\right): e^{-i \frac{\chi}{2}}:\left(y_{i}+\varepsilon\right) D\left(\mathscr{B}_{0},-2 \pi, y_{i}\right)\right]\right\rangle^{0} \\
\equiv(2 \pi)^{-n / 2}\left\langle\prod_{i=1}^{n} *\left[: e^{i \frac{\chi}{2}}:\left(x_{i}\right) D\left(\mathscr{B}_{0}, 2 \pi, x_{i}\right): e^{-i \frac{\chi}{2}}:\left(y_{i}\right) D\left(\mathscr{B}_{0},-2 \pi, y_{i}\right)\right]\right\rangle^{0} \\
=\left(-\frac{i}{2 \pi}\right)^{n} \operatorname{det}\left(\frac{1}{x_{i}-y_{i}}\right)=\left\langle\prod_{j=1}^{n} b\left(x_{j}\right) c\left(y_{j}\right)\right\rangle=\left\langle\prod_{j=1}^{n} b\left(x_{j}\right) \prod_{i=n}^{1} c\left(y_{i}\right)\right\rangle
\end{gathered}
$$

provided $x_{1}^{0}<\ldots<x_{n}^{0}<y_{n}^{0}<\ldots<y_{1}^{0}$.

More generally,

$$
\begin{aligned}
& (2 \pi)^{-n / 2}\left\langle\prod_{i=1}^{n} *\left[: e^{i \frac{\chi}{2}}:\left(x_{i}\right) D\left(\mathscr{B}_{0}, 2 \pi, x_{i}\right): e^{-i \frac{\chi}{2}}:\left(y_{i}\right) D\left(\mathscr{B}_{0},-2 \pi, y_{i}\right)\right]\right\rangle^{0} \\
& \quad=\left\langle T\left[\prod_{j=1}^{n} b\left(x_{j}\right) c\left(y_{j}\right)\right]\right\rangle,
\end{aligned}
$$

where $T$ is Euclidean time-ordering, i.e. $T$ permutes the factors (the $b$ 's and $c$ 's) in the product in such a way that the 0 -(time-)components of their arguments are in an increasing order and multiplies by the sign of the permutation. Passing from $\mathscr{B}_{0}$ to another branch, $\mathscr{B}$, changes the sign of the left-hand side of (3.70), and thus corresponds to replacing $T$ by some other ordering.

We conclude that we may identify

and

$$
\left.\begin{array}{l}
b(x) \text { with }: e^{i \frac{\chi}{2}}:(x) D(\cdot, 2 \pi, x) \\
c(x) \text { with }: e^{-i \frac{\chi}{2}}:(x) D(\cdot,-2 \pi, x) .
\end{array}\right\}
$$

Similarly, one shows that

and

$$
\left.\begin{array}{ll}
\bar{b}(x) \text { corresponds to } & : e^{i \frac{\chi}{2}}:(x) D(\cdot,-2 \pi, x) \\
\bar{c}(x) \text { corresponds to }: e^{-i \frac{\chi}{2}}:(x) D(\cdot, 2 \pi, x) .
\end{array}\right\}
$$

Using (3.28) and (3.44), (3.46), it is easy to show that

$$
\lim _{y \rightarrow x}: e^{i \frac{\chi}{2}}:(y) D(\cdot,-2 \pi, y): e^{i \frac{\chi}{2}}:(x) D(\cdot, 2 \pi, x)=e^{i \theta}: e^{i \chi}:(x),
$$


for some phase $\theta$ depending on the direction in which $y$ tends to $x$. [Using the notion of normal ordering introduced in (3.52), it follows that

$$
\left.\lim _{y \rightarrow x} N\left(: e^{i \frac{\chi}{2}}:(y) D(\cdot,-2 \pi, y): e^{i \frac{\chi}{2}}:(x) D(\cdot, 2 \pi, x)\right)= \pm i: e^{i \chi}:(x) .\right]
$$

Similarly,

$$
\lim _{y \rightarrow x}: e^{-i \frac{\chi}{2}}:(y) D(\cdot,-2 \pi, x): e^{-i \frac{\chi}{2}}:(x) D(\cdot, 2 \pi, x)=e^{-i \theta}: e^{-i \chi}:(x) .
$$

Formulas (3.73) and (3.74) reproduce (3.61).

We define spin- and disorder fields by

$$
\left.\begin{array}{cc}
\sigma^{ \pm}(x)=: e^{ \pm i \frac{x}{2}}:(x), & \sigma(x)=\frac{1}{2}\left(\sigma^{+}(x)+\sigma^{-}(x)\right), \\
\mu^{ \pm}(x)=D(\cdot, \pm 2 \pi, x), & \mu(x)=\frac{1}{2}\left(\mu^{+}(x)+\mu^{-}(x)\right) .
\end{array}\right\}
$$

One now easily verifies that $\sigma$ and $\mu, \sigma$ and $\psi$, and $\mu$ and $\psi$ all satisfy the "dual algebra" (2.44). Our formalism permits us to compute arbitrary correlation (Euclidean Green) functions of spin- and disorder fields.

The fields $\sigma$ and $\mu$ are the spin- and disorder fields of a theory of free, massless Dirac fermions. It is well known that the two-dimensional Ising model is equivalent to a theory of free Majorana fermions. In order to pass from Dirac- to Majorana fermions, partition- and spin-/disorder correlation functions must be factorized: Let $\$$ be the covariant Dirac operator for a connection which is a sum of an abelian gauge field, $A$, whose curvature is concentrated in points $\left(x_{1}, \ldots, x_{2 n}\right)$ and a chiral gauge field, $A^{5}$. whose curvature is concentrated in the points $\left(y_{1}, \ldots, y_{2 m}\right)$. Then, for a proper choice of $A$ and $A^{5}$,

$$
\left\langle\prod_{i=1}^{2 n} \sigma\left(x_{i}\right) \prod_{j=1}^{2 m} \mu\left(y_{j}\right)\right\rangle=\left[\frac{\operatorname{det}(\not+m)}{\operatorname{det}(\not \partial+m)}\right]_{\mathrm{ren}} .
$$

For $m=0$, this is an easy consequence of our formalism. The passage from Diracto Majorana-fermions consists in taking the square root of (3.76). This remark, together with (3.75), permits us to explicitly calculate the correlation functions of the critical Ising model, $(m=0)$. This provides a basis to the approach in [23].

One natural problem is to extend our formalism to Riemann surfaces of arbitrary genus. This problem has been solved in a somewhat abstract and indirect way in [22]. It might be useful to have a more concrete approach to these matters, using functional integration. The identification of $N(b c)$ with $\frac{1}{2 \pi} \partial \chi$ and of $N(\bar{b} \bar{c})$ with $\frac{1}{2 \pi} \bar{\partial} \chi$, where $\chi$ is a Gaussian random field over the Riemann surface with covariance given by the Green function of the Laplacian, is valid in general. What one needs, in addition, is an analogue of Cauchy's identity (3.59) for arbitrary Riemann surfaces.

Our formalism is very useful to explicitly describe some representations of the Virasoro algebra, for $c \geqq 1$, and of current (Kac-Moody) algebra in terms of 
functional integration over free, massless boson- and parafermion fields. These fields are "non-local" in the sense that they satisfy intermediate statistics, and their Euclidean Green functions therefore have non-trivial monodromy which yields non-trivial representations of the pure braid groups, (the Burau representations). But these matters go beyond the scope of the present paper.

Finally, our formalism can be used to recover the standard results concerning the Thirring model in a purely Euclidean setting. We mention this only because it will be useful in the next two sections. The action of the massless Thirring model is given by

$$
S(\bar{\psi}, \psi)=\int d^{2} x\left\{(\bar{\psi}, \not \psi \psi)(x)+\kappa j_{\mu}(x) j^{\mu}(x)\right\},
$$

with

$$
j^{\mu}=N\left(\bar{\psi} \gamma^{\mu} \psi\right)
$$

This model is bosonized by substituting in our formulas $d \mu^{0}(\chi)$ by $d \mu^{\kappa}(\chi)$, where $d \mu^{\kappa}$ is the Gaussian measure with mean 0 and covariance

$$
2\left(-\frac{1}{8 \pi} \Delta+\frac{\kappa}{4 \pi^{2}} \Delta\right)^{-1}=4 \pi\left(1-\frac{2 \kappa}{\pi}\right)^{-1}(-\Delta)^{-1} .
$$

This can be justified easily by using Eqs. (3.15), (3.57); (see also [14, 15] and refs. given there). By rescaling $\chi, \chi \rightarrow \beta \chi$, with $\beta=\left(1-\frac{2 \kappa}{\pi}\right)^{-1 / 2}$, we can express the theory in terms of $d \mu^{0},: e^{ \pm i \beta \chi}$, etc.

\section{Solitons in the Two-Dimensional Sine-Gordon Theory}

As an application of the formalism in Sect. 3, we now sketch the quantization of solitons in the two-dimensional sine-Gordon theory from the Euclidean point of view. This serves to elaborate on the Bose-Fermi equivalence already encountered in the last two sections and introduce some ideas that will be useful in Sect. 5.

The Euclidean field of the sine-Gordon model is denoted by $\chi$. The Euclidean action is given by

$$
S(\chi)=\int d^{2} x\left\{\frac{1}{8 \pi}:(\nabla \chi)^{2}:(x)+\lambda: \cos \beta \chi:(x)\right\},
$$

where Wick order, :- :, is defined with respect to the free field Gaussian measure $d \mu^{0}(\chi)$ with mean 0 and covariance $4 \pi(-\Delta+1)^{-1} ; \lambda$ is a real parameter and $\beta>0$. (We shall constrain $\beta$ to be $\leqq 1$.) The vacuum sector of this model can be reconstructed from the functional measure

$$
d \mu(\chi)=\frac{1}{Z} e^{-S(\chi)} D \chi_{0},
$$

where $D \chi_{0}$ is a formal Lebesgue measure defined as in (3.25).

The measure $d \mu$ has been constructed rigorously in [12], for all $\beta \leqq 1$. The methods of [13] can presumably be adapted to prove that the theory has a positive 
mass gap, at least for $\beta$ small enough. For $\beta=1$, the theory is equivalent to free, massive Dirac fermions, while, for $\beta \neq 1$, it is equivalent to the massive Thirring model, (see end of Sect. 3). The renormalization theory, for $1<\beta<2$, is discussed in [16], and, for $\beta>2$, the theory is expected to be equivalent to a free, massless Bose field. For the lattice theory this is the celebrated Kosterlitz-Thouless phase of the two-dimensional Coulomb gas which has been exhibited rigorously in [17]. We limit our discussion to the range $\beta \leqq 1$, as this suffices for the applications in Sect. 5.

The action $S(\chi)$ defined in (4.1) has a discrete symmetry group

$$
\chi(x) \rightarrow \chi(x)+\frac{2 \pi}{\beta} n, \quad n \in \mathbb{Z} .
$$

This symmetry is always spontaneously broken: For zero-Dirichlet b.c.

$$
\langle\Omega, \hat{\chi} \Omega\rangle=\int d \mu(\chi) \chi(x)=0
$$

Had we imposed zero-Dirichlet b.c. on the field $\chi(x)-\frac{2 \pi}{\beta} n, n \in \mathbb{Z}$, (i.e., formally, $\chi(x) \rightarrow \frac{2 \pi}{\beta} n$, as $\left.|x| \rightarrow \infty\right)$, we would have found

$$
\langle\Omega, \hat{\chi} \Omega\rangle=\frac{2 \pi}{\beta} n .
$$

[Here $\hat{\chi}$ is the relativistic quantum field corresponding to $\chi$.] Thus, the theory has a large vacuum degeneracy labelled by the integers, but, of course, all the vacuum sectors are isomorphic, (identical dynamics). It is therefore appropriate to view $: e^{i \beta \chi(x)}:$ and $(\nabla \chi)(x)$ as the basic fields of the theory which do not depend on the choice of vacuum, or b.c.

By analogy with the situation in the $\lambda \phi_{2}^{4}$-model, the vacuum degeneracy described above ought to give rise to soliton sectors the construction of which should be similar to that in $\lambda \phi_{2}^{4}$ and will now be outlined.

The soliton bundle for the sine-Gordon model can be identified with some soliton bundle $E_{\Phi}$ as defined in the previous section. The requirement on $E_{\Phi}$ is that the action density $S(\tilde{\chi})(x)$ obtained by substituting $\chi$ by a section $\tilde{\chi}$ of $E_{\Phi}$ in $(\overline{4} .1)$ and $\nabla$ by the covariant derivative $\nabla_{\Phi}$, is globally projectable.

The term $\cos \beta \tilde{\chi}$ is globally projectable iff $\tilde{\chi}$ is a section of a bundle $E_{\Phi}$ characterized by the flux numbers

$$
\Phi_{w_{l}}=\Phi_{i} \in \frac{2 \pi}{\beta} \mathbb{Z}
$$

This immediately follows from the fact that the gauge transformation

$$
\chi(x) \rightarrow \chi(x)+\frac{2 \pi}{\beta} n(x), \quad n(x) \in \mathbb{Z},
$$

leaves $\cos \beta \chi$ invariant.

To construct soliton Green functions for the sine-Gordon model we thus choose a function $\tilde{\alpha}$ on $\tilde{M}_{\underline{w}}$ defining a section of $E_{w,}$. with $\Phi$ as in (4.5), and define a 
covariant action by

$$
\begin{aligned}
S(\chi) & =\int_{M_{w}} d^{2} x\left\{\frac{1}{8 \pi}:\left(\nabla_{\Phi} \tilde{\chi}\right)^{2}:(x)-\lambda: \cos \beta \tilde{\chi}:(x)\right\} \\
& =\int_{M_{w}} d^{2} x\left\{\frac{1}{8 \pi}:\left(\nabla \chi+A_{\underline{\Phi}}\right)^{2}:(x)-\lambda: \cos \beta(\chi+\tilde{\alpha}):(x)\right\} \\
& \equiv S(\chi, \tilde{\alpha}) .
\end{aligned}
$$

We then formally define

$$
d \mu_{\tilde{\alpha}}(\chi)=\left[\frac{1}{Z} e^{-S(\chi, \tilde{\alpha})}\right]_{\mathrm{ren}} D \chi_{0} .
$$

A mathematical definition of (4.8) is achieved by taking the limit, as $\Lambda \nearrow \mathbb{R}^{2}$, of the characteristic functionals of the measures

where

$$
d \mu_{\tilde{\alpha}, \Lambda}(\chi) \equiv \frac{1}{Z_{\Lambda}} e^{V_{\tilde{\alpha}, \Lambda}(\chi)} d \mu_{A}^{0}(\chi)
$$

$$
V_{\tilde{\alpha}, \Lambda}(\chi)=\lambda \int_{\Lambda} d^{2} x: \cos \beta(\chi+\tilde{\alpha}):(x)
$$

and

$$
Z_{\Lambda}=\int d \mu^{0}(\chi) e^{V_{\Lambda}(\chi)}, \quad(\tilde{\alpha} \equiv 0) .
$$

The measure $d \mu_{A}^{0}$ is the one constructed in (3.46), Sect. 3, with $\Phi$ satisfying (4.5). The limiting measure, $d \mu_{\tilde{\alpha}}(\chi)$, as $\Lambda \nearrow \mathbb{R}^{2}$, will also be written as

$$
\frac{1}{Z} e^{V_{\tilde{\alpha}}(\chi)} d \mu_{A}^{0}(\chi)
$$

Gauge-invariant mixed meson-soliton Green functions of the sine-Gordon theory are now defined by

$$
\begin{gathered}
G_{s}^{(n, n, m)}\left(x_{1}, \ldots, x_{n}, y_{1}, \ldots, y_{n} ; z_{1}, \varepsilon_{1}, \ldots, z_{m}, \varepsilon_{m}\right) \\
\quad=\frac{1}{Z} \int e^{V_{\tilde{\alpha}}(\chi)} \prod_{j=1}^{m}: e^{i \varepsilon_{j} \beta(\chi+\tilde{\alpha})}:\left(z_{j}\right) d \mu_{A}^{0}(\chi),
\end{gathered}
$$

where $\varepsilon_{i}= \pm 1, i=1, \ldots, n$, and $\tilde{x}$ defines a section of $E_{\underline{\Phi}}, \Phi=\Phi_{\underline{w}}$, with $\underline{w}=\left(x_{1}, \ldots, x_{n}\right.$, $\left.y_{1}, \ldots, y_{n}\right)$ and $\Phi_{x_{i}}=\frac{2 \pi}{\beta}, \Phi_{y_{i}}=-\frac{2 \pi}{\beta}, i=1, \ldots, n$.

As the notation on the left-hand side of (4.10) indicates, the functional integral on the right-hand side of (4.10) only depends on our choice of $\underline{w}$ and $\left\{\Phi_{x_{i}}, \Phi_{y_{l}}\right\}_{i=1}^{n}$, but not on an explicit choice of $\tilde{\alpha}$. To see this, let $\alpha_{0}$ be a globally defined function on $M_{\underline{w}}$. From its definition (3.46) it follows easily that

$$
d \mu_{A+\nabla \alpha_{0}}^{0}(\chi)=d \mu_{A}^{0}\left(\chi+\alpha_{0}\right) .
$$

If, on the right-hand side of (4.10), we replace $\tilde{\alpha}$ by $\tilde{\alpha}+\alpha_{0}$, use (4.11) and then change variables, $\chi^{\prime}=\chi+\alpha_{0}$, under the integral our claim follows. 
In particular, we can consider the limiting case obtained by choosing $\tilde{\alpha}=\alpha_{h}$ and

$$
\alpha_{0}=* V^{*} \Delta^{-1} j
$$

where $\frac{\beta}{2 \pi} j$ is an integer current satisfying

$$
\nabla \cdot j=\sum_{i} \Phi_{i} \delta_{w_{i}}
$$

Then by (3.37)

$$
d \mu_{A_{h}+\nabla \alpha_{0}}^{0}=d \mu_{j}^{0}
$$

and

$$
V_{\alpha_{h}+\alpha_{0}}=V_{0}=V, \quad: e^{i \varepsilon \beta\left(\chi+\alpha_{h}+\alpha_{0}\right)}:=: e^{i \varepsilon \beta \chi}:,
$$

so that the only dependence on the connection is now in $d \mu_{j}^{0}$. Furthermore, by performing a gauge transformation

$$
\alpha_{0} \rightarrow \alpha_{0}+\frac{2 \pi}{\beta} n \chi_{B}
$$

where $B$ is an arbitrary region in $M_{\underline{w}}$ and $n \in \mathbb{Z}$, we change $j$ into a new 1 -current $j^{\prime}$ such that $\frac{\beta j^{\prime}}{2 \pi}$ is an integer current and $j^{\prime}$ still satisfies (4.13). The construction of Green functions with $d \mu_{j}^{0}$ is the strict continuum analogue of the lattice construction described in [2].

We remark that the couple of forms $\left(A_{h},\left.\alpha_{0}\right|_{M_{w} \backslash \operatorname{supp} j}\right)$ defines an AllendoerferEells 1-form in $M_{w}$, [18].

As shown in Sect. 3,

and

$$
d \mu_{A_{h}}^{0}(\chi)=e^{-\frac{1}{8 \pi}\left\langle A_{h}, A_{h}\right\rangle_{\mathrm{reg}}} d \mu^{0}(\chi)
$$

$$
e^{-\frac{1}{8 \pi}\left\langle A_{h}, A_{h}\right\rangle_{\mathrm{reg}}}=\exp \left[-\frac{1}{16 \pi^{2}} \sum_{i \neq j} \Phi_{i} \Phi_{j} \ln \frac{1}{\left|w_{i}-w_{j}\right|}\right]=\mid \operatorname{det}\left(\frac{1}{x_{i}-y_{j}}\right)^{\frac{1}{2 \beta^{2}}},
$$

where $\underline{w}$ and $\Phi$ are as in (4.10).

It is also easy to check that

$$
\left|\frac{1}{Z} \int e^{V_{\tilde{\alpha}}(\chi)} \prod_{j=1}^{m}: e^{i \varepsilon_{j} \beta(\chi+\tilde{\alpha})}:\left(z_{j}\right) d \mu^{0}(\chi)\right| \leqq \frac{1}{Z} \int e^{V(\chi)} \prod_{j=1}^{m}: e^{i \varepsilon_{j} \beta \chi}:\left(z_{j}\right) d \mu^{0}(\chi),
$$

see [12] for the relevant calculations. Hence, by (4.17-4.19)

$$
\left|G_{s}^{(n, n, m)}\left(x_{1}, \ldots, \varepsilon_{m}, z_{m}\right)\right| \leqq\left|\operatorname{det}\left(\frac{1}{x_{i}-y_{j}}\right)\right|^{\frac{1}{2 \beta^{2}}} G^{(0,0, m)}\left(z_{1}, \varepsilon_{1}, \ldots, z_{m}, \varepsilon_{m}\right) .
$$

Results in [12] and bound (4.20) prove that the Green functions $G_{s}^{(n, n, m)}$ are well defined distributions for $\beta \leqq 1$ and that $G^{(n, n, 0)}$ satisfies cluster properties. 
It is not hard to convince oneself that the Green functions $\left\{G_{s}^{(n, n, m}\right\}$ satisfy the Osterwalder-Schrader axioms; (O.S. positivity follows, using the measure $d \mu_{j}^{0}$ defined in (4.14), for suitable choices of $j)$. The local soliton fields $s(x), \bar{s}(y)$ reconstructed from $\left\{G_{s}^{(n, n, m)}\right\}$ are Bose fields. since

$$
G_{s}^{(n, n, m)}\left(x_{1}, \ldots, x_{n}, y_{1}, \ldots, y_{n} ; z_{1}, \varepsilon_{1}, \ldots, z_{m}, \varepsilon_{m}\right)
$$

is totally symmetric under exchanging the $x$-arguments and the $y$-arguments among themselves, and its phase depends continuously on $\underline{x}, \underline{y}$ and $\underline{z}$ at noncoinciding arguments.

But, as follows from the discussion in Sect. 3, there are other soliton fields, $b\left(x_{i}\right)$, $c\left(y_{i}\right)$ (and $\left.\bar{b}, \bar{c}\right)$ which are chiral Dirac Fermi fields. They can be reconstructed from the following Green functions: We choose $\tilde{\alpha}$ as in (4.10).

It is mathematically consistent to set

$$
\tilde{\alpha}_{\Phi_{x_{i}}}\left(x_{i}\right)=\tilde{\alpha}_{\Phi_{y_{t}}}\left(y_{i}\right)=0 \text {. }
$$

Then the fermionic Green functions are defined by

$$
\begin{aligned}
\widetilde{G}_{s}^{(n, n, m)}\left(x_{1}, \ldots, x_{n}, y_{1}, \ldots, y_{n} ; z_{1}, \varepsilon_{1}, \ldots, z_{m}, \varepsilon_{m}\right) \\
=\frac{1}{Z} \int e^{V_{\tilde{\alpha}}(\chi)} \prod_{j=1}^{n}\left(: e^{i \frac{\beta}{2}(\chi+\tilde{\alpha})}:\left(x_{j}\right): e^{-i \frac{\beta}{2}(\chi+\tilde{\alpha})}:\left(y_{j}\right)\right) \\
\quad \times \prod_{j=1}^{m}: e^{i \varepsilon_{\jmath} \beta(\chi+\tilde{\alpha})}:\left(z_{j}\right) d \mu_{A}^{0}(\chi) .
\end{aligned}
$$

[To verify Fermi statistics of the fields $b$ and $c$ reconstructed from (4.21), it is convenient to work with $d \mu_{j}^{0}$. The argument is then very similar to that used to verify the dual algebra (2.44) in the $\lambda \phi_{2}^{4}$-model; see (2.50).]

It is not hard to show that the fields $s$ and $b$ carry topological charge $Q=1$, (i.e. $[Q, s]=[Q, b]=1$, while $\bar{s}$ and $c$ carry topological charge $Q=-1$. Here

$$
Q=\frac{\beta}{2 \pi} \int_{-\infty}^{+\infty}\left(\frac{\partial}{\partial x^{1}} \hat{\chi}\right)\left(0, x^{1}\right),
$$

where $\hat{\chi}$ is the quantum field corresponding to the Euclidean field $\chi$. The proofs are very similar to that in Sect. 2.5, (2.51)-(2.55). One sees easily that, in the sineGordon model, the topological charge is additive. There are physical states of arbitrary charge $q \in \mathbb{Z}$.

Finally, we remark that, for $\beta<1$ small enough, it appears quite straightforward, though technically involved, to establish exponential cluster decomposition properties of the Green functions $\left\{G_{s}^{(n, n, m)}\right\}$, or $\left\{\widetilde{G}_{s}^{(n, n, m)}\right\}$, and to prove that the field $: \sin \beta \chi$ : couples the vacuum to a stable, neutral one-particle state, while $s, b$ and $\bar{s}, c$ couple the vacuum to stable one-particle states of charge \pm 1 . (In the lattice approximation, this can be verified by applying the methods of $[2,19]$.)

\section{Solitons with Fractional Fermion Number}

In this section we combine the techniques of Sects. 2-4 to discuss a class of twodimensional interacting boson-fermion models, including the pseudoscalar Yukawa model (with Thirring term). 
The Euclidean fields of the model are the (Dirac) fermion fields $\psi, \bar{\psi}$ and the real Bose field $\Phi$. The action is given by

$$
\begin{gathered}
S(\Phi, \psi, \bar{\psi}) \equiv S_{B}(\Phi)+S_{F}(\Phi, \psi, \bar{\psi}), \\
S_{B}(\Phi)=\int\left\{\frac{1}{2}:(\nabla \Phi)^{2}:(x)+\frac{\lambda}{4}:\left(\Phi^{2}-\xi\right)^{2}:(x)\right\} d^{2} x, \\
S_{F}(\Phi, \psi, \bar{\psi})=\int\left\{: \bar{\psi} \phi \psi:(x)-N\left[\bar{\psi}\left(m_{f}+i g \gamma_{5} \Phi\right) \psi\right](x)+\left(\kappa j_{\mu} \mu^{\mu}\right)(x)\right\} d^{2} x,
\end{gathered}
$$

where the last term is the Thirring term; (see end of Sect. 3).

A rigorous construction of the Q.F.T. corresponding to the formal action (5.1), with $\kappa=0$, has been achieved in $[24,25]$.

Jackiw and Rebbi [26] considered the model with $m_{f}=0$ in the approximation where $\Phi$ is treated as an external field. This serves as a starting point for a W.K.B. expansion.

They found that the Dirac equation for the field $\psi$ in the background of a static kink of the $\phi_{2}^{4}$ model exhibits a normalizable zero mode, $\psi_{0}$. (In this context, the existence of $\psi_{0}$ was later derived from the index theorem [27].) On the basis of the W.K.B. approximation, they argued that the one-soliton state of the quantum $\phi_{2}^{4}$ model gives rise, in the quantum field theory described by (5.1), to a doubly degenerate soliton state, corresponding to $\psi_{0}$ being occupied or empty. A computation of the fermion number, $n_{f}$, of such solition states, based on a mode expansion of $\psi$, yields the remarkable result

$$
n_{f}=+\frac{1}{2},-\frac{1}{2} \text {. }
$$

Subsequently, the model was studied also for $m_{f} \neq 0$, with the same methods [28]. As a result an argument was put forward supporting the existence of soliton states with fermion number given by

$$
n_{f}= \pm \pi^{-1} \arctan \left(\frac{g \sqrt{\xi}}{m_{f}}\right) .
$$

There are analogues of all these models in condensed matter physics with similar features. In particular, a model with solitons of fermion number $\pm \frac{1}{2}$ has been considered in connection with polyacetylene [29].

In this section we rigorously prove that, for a suitable range of parameters the fully quantized theory possesses soliton sectors which are eigenspaces of the fermion number operator corresponding to fractional eigenvalues. However, for $m_{f} \neq 0$, quantum corrections have to be added to the semi-classical result (5.3). These corrections disappear by symmetry, for $m_{f}=0$, and we recover (5.2).

We also prove (in the lattice approximation) that the soliton field operator maps the vacuum of the theory to a stable massive one-particle state; i.e. the Q.F.T. described by (5.1) really possesses particles of fractional fermion number.

To construct the Euclidean disorder field of the model from which a local soliton field operator can be reconstructed we need to know the symmetries of the action (5.1). They are most clearly displayed if we rewrite the fermion action, $S_{F}$, in terms of chiral fermi fields, $b, \bar{b}, c, \bar{c}$, as in Sect. 3 .

$$
\begin{aligned}
S_{F}(b, \bar{b}, c, \bar{c}, \Phi)= & \int d^{2} x\left\{b \partial c+\bar{b} \bar{\partial} \bar{c}-m_{f} N(\bar{b} b+c \bar{c})\right. \\
& \left.-g N(\bar{b} b-c \bar{c}) \Phi(x)+\kappa[N(\bar{b} \bar{c}-c b)]^{2}+\kappa[N(\bar{b} \bar{c}+c b)]^{2}\right\} .
\end{aligned}
$$


The total action is invariant under the transformation

$$
\Phi \rightarrow-\Phi, \quad b \leftrightarrow \bar{c}, \quad \bar{b} \leftrightarrow c .
$$

The model can be bosonized using the rules of Sect. 3. Denoting by $\chi$ a real Bose field used in the bosonization of (5.4), the bosonized action reads

$$
S_{F}=\int\left\{\frac{1}{8 \pi}:(\nabla \chi)^{2}:(x)-m_{f}: \cos \beta \chi:(x)-g: \sin \beta \chi:(x) \cdot \Phi(x)\right\} d^{2} x,
$$

where

$$
\beta=\left(1-\frac{2 \kappa}{\pi}\right)^{-1 / 2}
$$

This action displays a $\mathbb{Z}_{2}$-symmetry which is the bosonized version of the transformation (5.5):

$$
\Phi \rightarrow-\Phi, \quad \chi \rightarrow-\chi,
$$

and a $\mathbb{Z}$-symmetry, corresponding to the transformation

$$
\Phi \rightarrow \Phi, \quad \chi \rightarrow \chi+\frac{2 \pi}{\beta} n, \quad n \in \mathbb{Z} .
$$

The classical equations of motion have two minimizing solutions:

$$
\phi_{c}= \pm \sqrt{\xi}, \quad \chi_{c}=\frac{1}{\beta} \arctan \left(\frac{g \phi_{c}}{m_{f}}\right) .
$$

The expression for $\chi_{c}$ is easily obtained by first setting $\phi=\phi_{c}$ and, then minimizing the action. We obtain

$$
\frac{\delta}{\delta \chi}\left[-m_{f} \cos \beta \chi-g \sin \beta \chi \phi_{c}\right]=0
$$

hence

$$
\tan \left(\beta \chi_{c}\right)=\frac{g \phi_{c}}{m_{f}}
$$

From the functional integrals with zero Dirichlet b.c. for $\phi-\phi_{c}$ and $\chi-\chi_{c}$ at infinity $\left(\phi_{c}= \pm \sqrt{\xi}\right)$ we can reconstruct two physical vacua, $\Omega_{ \pm}$. By a Peierls argument [13], or by means of a combined contour-cluster expansion [25], one can prove that the two vacuum states $\left\langle\Omega_{ \pm},(\cdot) \Omega_{ \pm}\right\rangle$are distinct, for $g$ large enough, $m_{f}$ and/or $\lambda$ large, and the symmetry (5.7) (equivalent to (5.5)) is spontaneously broken. This gives rise to a non-trivial superselection structure in the form of soliton (kink- and antikink-)sectors. The construction and analysis of these sectors is the main theme of this section.

The additive symmetry (5.8) is always spontaneously broken, just as in the sineGordon model, and the corresponding soliton sectors can be constructed as in Sect. 4. One version of the local soliton fields arising in this construction is simply the bosonized form of the Fermi fields $\psi, \bar{\psi}$ of the original model.

More interesting are the soliton sectors and soliton fields arising from the breaking of the symmetry (5.5) on which we focus our attention in the following. 
First we outline their construction in fermionic language, subsequently we consider the same problem in bosonic language. Let $E_{\psi}, E_{\bar{\psi}}$ be two twisted Euclidean spinor bundles [30] with base space

$$
M_{\underline{w}}=\mathbb{R}^{2} \backslash\left\{w_{1}, \ldots, w_{k}\right\}
$$

and structure group $\mathbb{Z}_{2}$. We define a flat connection on these bundles in terms of its holonomy: If $\omega$ is a loop in $M_{\underline{w}}$, then, in the chiral basis described in Sect. 3, the holonomy, $U(\omega)$, is given by

$$
U(w)=\left(\begin{array}{ll}
0 & 1 \\
1 & 0
\end{array}\right)^{\sum_{i=1}^{k} n\left(\omega, w_{i}\right)},
$$

where $n\left(\omega, w_{i}\right)$ is the winding number of $\omega$ with respect to $w_{i} ; U(\omega)$ is defined by left action on the sections of $E_{\psi}$, locally of the form $\psi=\left(\begin{array}{l}b \\ \bar{c}\end{array}\right)$, and by right action on the sections of $E_{\bar{\psi}}$ which are locally of the form $\bar{\psi}=(\bar{b}, c)$.

Let $E_{\Phi}$ denote the soliton bundle associated with the scalar field $\Phi$ which we introduced in Sect. 2.

The soliton bundle for the pseudoscalar Yukawa model is now defined as the fiber product of $E_{\Phi}, E_{\psi}$ and $E_{\bar{\psi}}$, i.e. as the bundle over $M_{\underline{w}}$ whose fiber is the cartesian product of the fibers of $E_{\Phi}, E_{\psi}$ and $E_{\bar{\psi}}$. The point is now that the covariant action density of the pseudoscalar Yukawa model (see (5.1)) is a single-valued function on $M_{w}$, for arbitrary sections $\psi, \bar{\psi}$ and $\Phi$ of the soliton bundle defined above. This is a consequence of the symmetry (5.5). If $k$ is even then there are plenty of sections of finite total action.

It is now clear how to construct soliton Green functions directly in the fermionic language. We omit a detailed analysis and, instead, turn to our analysis of the soliton sectors of the bosonized pseudoscalar Yukawa model (although the fermionic language might perhaps be more natural).

Let $E_{\chi}$ be a soliton bundle, with sections denoted by $\chi(\cdot)$, which is an isomorphic copy of the bundle $E_{\Phi}$ constructed in Sect. 2 . Then a soliton bundle for the bosonized pseudoscalar Yukawa model is the fiber product of $E_{\Phi}$ and $E_{\chi}$, with base space $M_{\underline{x}} \equiv \mathbb{R}^{2} \backslash\left\{x_{1}, \ldots, x_{2 n}\right\}$. Let $\underline{\gamma}$ be a family of paths in $M_{\underline{x}}$ with $\partial \underline{\gamma}$ $=\left\{x_{1}, \ldots, x_{2 n}\right\}$. Let $d \mu_{\gamma}^{0}$ be the Gaussian measure with mean 0 and covariance $\left(-\Delta_{\gamma}+1\right)^{-1}$ constructed in Sect. 2 ; see Eqs. (2.36)-(2.39).

Then the mixed order-disorder correlation functions for the bosonized pseudoscalar Yukawa model are defined by

$$
\begin{aligned}
& G_{s, \pm}\left(x_{1}, \ldots, x_{2 n} ; y_{1}, \ldots, y_{m} ; z_{1}, \ldots, z_{p} ; \ldots\right) \\
& \quad \equiv z(\partial \underline{\gamma})^{2} \frac{\iint d \mu_{\gamma}^{0}(\Phi) d \mu_{\underline{\gamma}}^{0}(\chi) e^{-V_{ \pm}(\Phi, \chi)} \prod_{i=1}^{m} \Phi_{\underline{\gamma}}\left(y_{i}\right) \prod_{j=1}^{p}: \cos \beta \chi:\left(z_{j}\right) \ldots}{\iint d \mu^{0}(\Phi) d \mu^{0}(\chi) e^{-V_{ \pm}(\Phi, \chi)}},
\end{aligned}
$$

where

$$
\begin{aligned}
V_{ \pm}(\Phi, \chi)= & {\left[\int d ^ { 2 } x \left\{\frac{\lambda}{4}:\left(\Phi^{2}-\xi\right)^{2}:(x)-\frac{1}{2}: \Phi^{2}:(x)-\frac{1}{2}: \chi^{2}:(x)\right.\right.} \\
& \left.\left.-m_{f}: \cos \beta \chi:(x)-g: \sin \beta \chi:(x) \Phi(x)\right\}\right]_{ \pm},
\end{aligned}
$$


with + or - b.c. at infinity: [see remark after (5.9)]. The dots in (5.11) stand for additional functionals of $\chi$, like $: \sin \beta \chi_{\gamma}: \partial_{\mu} \chi_{\gamma}$. We note that $d \mu_{\gamma}^{0}(\Phi) d \mu_{\gamma}^{0}(\chi)$ is a Gaussian measure on a space of section distributions of the soliton bundle over $M_{\underline{x}}$ for the bosonized pseudoscalar Yukawa model.

Following the strategy described in Sect. 2 (see also $[2,9]$ ) one can prove that the Green functions $G_{s, \pm}$ satisfy Osterwalder-Schrader axioms. The property most difficult to establish is clustering. A proof of clustering would require a "cluster expansion" combining the expansions in $[5 \mathrm{~b}, 13,9]$. It would converge for small $\beta$ and $\lambda$ and $\xi=0\left(\lambda^{-1}\right)$. Alternatively, an expansion, like the one in [25], could be applied directly to the fermionic model and would converge for $\kappa=0$ and $m_{f}, g$ large. For the lattice approximation to the bosonized pseudo-scalar Yukawa model, clustering and many other properties are easily established with the help of a "Combined Low- and High-Temperature Expansion" $[2,31]$. (Of course, this expansion which is quite crude does not converge uniformly in the lattice spacing.) Some basic steps of that expansion are outlined in the appendix.

The techniques used to prove clustering show that all correlation functions with an odd number of $x$-arguments, obtained by removing $x_{2 n}$ to infinity, vanish, and the Hilbert space of physical states, $\mathscr{H}$, obtained via O.S. reconstruction (with + or - b.c.) decomposes into two orthogonal sectors: $\mathscr{H}_{+} \oplus \mathscr{H}_{s}, \mathscr{H}_{-} \oplus \mathscr{H}_{\bar{s}}$, respectively. The soliton field operators, reconstructed from the Green functions (5.11), map $\mathscr{H}_{+}$onto $\mathscr{H}_{s}$ and $\mathscr{H}_{-}$to $\mathscr{H}_{\bar{s}}$.

We now compute the fermion number of the soliton states. The matrix elements of the fermion number operator, $Q_{F}$, are given by

$$
\left\langle F\left|Q_{F}\right| G\right\rangle=\left\langle\theta F\left(\int_{-\infty}^{+\infty} j^{0}\left(x^{0}, x^{1}\right) d x^{1}\right) G\right\rangle,
$$

where $|F\rangle$ is a vector in $\mathscr{H}$ and $F$ the corresponding function of the fields $\Phi, \psi, \bar{\psi}$ in $\widetilde{F}+\left[\right.$ see Sect. 2, Eq. (2.49)] and $j^{0}\left(x^{0}, x^{1}\right)$ is the 0 -component of the fermion current.

In the presence of a singular external $\mathbb{Z}_{2}$-gauge field [gauging the symmetry (5.7)] with support in $\underline{\gamma}$, the (gauge-invariant) fermion current is defined by

$$
j_{\underline{\underline{\gamma}}}^{\mu}(x)=\lim _{\varepsilon \rightarrow 0} N\left(\bar{\psi}_{x+\varepsilon} \gamma^{\mu} U_{\underline{\gamma}}\left(\Gamma_{x, \varepsilon}\right) \psi_{x-\varepsilon}\right),
$$

where $U_{\gamma}\left(\Gamma_{x, \varepsilon}\right)$ denotes the parallel transport along the curve $\Gamma_{x, \varepsilon}$ joining $x-\varepsilon$ to $x+\varepsilon$ which is given, in the chiral basis, by

$$
\left(\begin{array}{ll}
0 & 1 \\
1 & 0
\end{array}\right)^{n\left(\Gamma_{x, \varepsilon}, \gamma\right)}
$$

Here $n\left(\Gamma_{x, \varepsilon}, \underline{\gamma}\right)$ denotes the number of intersections of $\underline{\gamma}$ and $\Gamma_{x, \varepsilon}$.

Using the techniques of Sect. 3, we may identify

$$
j_{\underline{\gamma}}^{0}\left(x^{0}, x^{1}\right) \leftrightarrow \frac{\beta}{2 \pi} \frac{\partial}{\partial x_{1}} \chi_{\underline{\gamma}}\left(x^{0}, x^{1}\right) .
$$


Hence, by following the steps which lead from (2.51) to (2.55), we immediately obtain:

$$
\begin{aligned}
& \left\langle e^{-\varepsilon H} S\left(0, x^{1}\right) F\left|Q_{F} e^{-\varepsilon^{\prime} H} S\left(0, y^{\prime}\right)\right| G\right\rangle \\
& \quad=2 \frac{\beta}{2 \pi}\left\langle\Omega_{(-)}|\chi| \Omega_{(-)}\right\rangle\left\langle e^{-\varepsilon H} S\left(0, x^{1}\right) F \mid e^{-\varepsilon^{\prime} H_{H}} S\left(0, y^{\prime}\right) G\right\rangle,
\end{aligned}
$$

where $|F\rangle,|G\rangle \in \mathscr{H}_{(-)}^{+}$

Using the fact that linear combinations of states of the from $e^{-\varepsilon H} s\left(0, x^{1}\right)|F\rangle$ are dense in $\mathscr{H}_{(\bar{s})}^{s}$, this shows that $\mathscr{H}_{(\bar{s})}$ is an eigenspace of $Q_{F}$ corresponding to the eigenvalue

$$
n_{F}^{s}=\frac{\beta}{\pi}\langle\chi\rangle_{+}, \quad n_{F}^{\bar{s}}=\frac{\beta}{\pi}\langle\chi\rangle_{-} .
$$

A semiclassical expansion of the form developed in $[5 b, 13]$ could be used to show that if $\lambda \xi$ is large, $\beta$ is small and $\beta^{2} g \sqrt{\xi}$ is large,

$$
\langle\chi\rangle_{ \pm}= \pm \frac{1}{\beta} \arctan \left(\frac{g \sqrt{\xi}}{m_{f}}\right)+\text { quantum corrections. }
$$

This is essentially the result of [28]. [To be precise, one can most probably prove rigorously, using the methods of $[5 b, 13]$, that the map from parameter space, $\left(\lambda, \xi, \beta, g, m_{f}\right)$, to $\frac{\beta}{\pi}\langle\chi\rangle+$ is smooth and non-constant, with derivatives given by perturbation theory around the semiclassical result. $]$

In conclusion, in the broken symmetry phase the model (5.1) exhibits soliton states with fractional fermion number depending continuously on the bare parameters of the model (in a certain region of bare parameters). The particle structure of the model can be analyzed, too, at least within the lattice approximation to the bosonized model: Combining the methods sketched in the Appendix with those in [19] one can prove that the soliton field, $s(x)$, couples the vacuum $\Omega_{+}$ to a stable, massive one-particle state which carries fractional fermion number.

Our findings can be summarized as follows:

1) In order to construct the soliton sectors of the $\lambda \phi_{2}^{4}$ model (see Sect. 2) in terms of Euclidean region functional integrals, we must twist the Euclidean $\Phi$-fields, as described in Sect. 2 . The resulting states in the soliton sectors are eigenstates of the topological charge,

$$
Q=\int_{-\infty}^{+\infty}\left(\partial_{1} \phi\right)\left(x^{0}, x^{1}\right) d x^{1}
$$

with eigenvalue $\pm 2\langle\phi\rangle_{+}$.

2) In the bosonized form of the pseudo-scalar Yukawa model, we must not only twist the $\Phi$-field but also the $\chi$-field, in order to construct soliton Green functions. This is required by the symmetry (5.7). The states reconstructed from the soliton 
Green functions (5.11) are eigenstates of the fermion number operator $Q_{F}$ with eigenvalue $\pm \frac{\beta}{\pi}\langle\chi\rangle_{+}$.

From such states we obtain physical states of fermion number $\pm \frac{\beta}{\pi}\langle\chi\rangle_{+}+n$, $n \in \mathbb{Z}$, by applying products of $\psi$-and/or $\bar{\psi}$ fields. The bosonized form of the $\psi$-and $\bar{\psi}$ fields of the bosonized pseudo-scalar Yukawa model is obtained by the construction described for the sine-Gordon model in Sect. 4. [The structure of these fields is connected with the symmetry (5.8) of the bosonized action which is always spontaneously broken.]

The superselection structure of the pseudo-scalar Yukawa model with $m_{f} \neq 0$ is thus described by a direct sum of superselection sectors, $\mathscr{H}_{i, n}, i=+,-, s, \bar{s}, n \in \mathbb{Z}$, the vacuum sectors corresponding to $\mathscr{H}_{ \pm, 0}$, and the sectors containing charged one particle states to $\mathscr{H}_{ \pm, \pm 1}, \mathscr{H}_{\bar{s} .0}, \mathscr{H}_{s, 0}$.

In the limit where $m_{f} \searrow 0$,

$$
n_{F}^{s} \rightarrow \frac{1}{2}, \quad \text { and } \quad n_{F}^{\bar{s}} \rightarrow-\frac{1}{2},
$$

(without any quantum corrections). This result is not only exact but rigorous and agrees with the one in [26] which was based on a semiclassical analysis. The reason why there are no quantum corrections in (5.16) is that when $m_{f}=0$ the model exhibits an additional symmetry:

$$
\Phi \rightarrow-\Phi, \quad \chi \rightarrow \chi+\frac{\pi}{\beta}
$$

from which it follows that

$$
\frac{\beta}{\pi}\langle\chi\rangle=\frac{1}{2}+n, \quad n \in \mathbb{Z} .
$$

3) One might ask whether the new symmetry (5.17) will give rise to additional soliton sectors which could not be obtained by the previous construction? We shall argue that this is actually not the case: The soliton sectors constructed above and the sectors constructed on the basis of the symmetry (5.17) - the construction combines the methods of Sect. 2 for $\Phi$ with the methods of Sects. 3, 4 for $\chi$ - are expected to be equivalent, and this can actually be proven quite easily in the lattice approximation. Roughly speaking, the argument for this runs as follows: Let $\mathscr{H}_{\sigma}$, $\mathscr{H}_{\bar{\sigma}}$ denote the soliton sectors and $\sigma, \bar{\sigma}$ the soliton fields reconstructed from soliton Green functions whose construction is based on soliton bundles with structure group given by (5.17). We propose to show that some states in $\mathscr{H}_{\sigma}$ have nonvanishing scalar product with some states in $\mathscr{H}_{s}$; (this is the basic step in proving that $\mathscr{H}_{\sigma}=\mathscr{H}_{s}$. . More precisely, we shall argue that

$$
\left\langle e^{-y^{0} H} \sigma\left(0, y^{1}\right) \Omega_{+}, e^{-x^{0} H} S\left(0, x^{1}\right) \Omega_{+}\right\rangle \neq 0,
$$

where $y^{0}$ and $x^{0}$ are positive. The scalar product on the left-hand side of (5.19) can be extracted from a four-point soliton Green function, $G(r y, r(y+a), x, x+a)$, where $a=\left(0, a^{1}\right)$, in the limit where $a^{1} \rightarrow \infty$. The symbol $r$ denotes reflection in the 
time-zero plane. According to our constructions in Sects. 2-4,

$$
\begin{aligned}
& \left\langle e^{-y^{0} H} \sigma\left(0, y^{1}\right) \Omega_{+}, e^{-x^{0} H} s\left(0, x^{1}\right) \Omega_{+}\right\rangle^{2}=\lim _{a^{1} \rightarrow \infty} G(r y, r(y+a), x, x+a) \\
& =\lim _{a^{1} \rightarrow \infty} \frac{1}{Z} \iint D_{E} \chi_{+} D_{E} \phi_{+} e^{-S(x, \phi)} \exp \int d^{2} x\left\{\frac{1}{8 \pi}\left[(\nabla \chi)^{2}(x)-\left(\nabla_{\gamma_{1}} \chi-\frac{\pi}{\beta} j_{2}\right)^{2}(x)\right]\right. \\
& \left.\quad+\frac{1}{2}\left[(\nabla \phi)^{2}(x)-\left(\nabla_{\gamma_{1} \gamma_{2}} \phi\right)^{2}(x)\right]\right\},
\end{aligned}
$$

where $\gamma_{1}$ and $\gamma_{2}$ are paths with $\partial \gamma_{1}=\{x, x+a\}, \partial \gamma_{2}=\{r y, r(y+a)\}$, and $j_{2}$ is a current of unit strength supported on $\gamma_{2}$. By gauge invariance, we can choose $\gamma_{1}$ and $\gamma_{2}$ to coincide except in compact discs around $\{x, r y\}$ and around $\{x+a$, $r(y+a)\}$, denoted by $D, D_{a}$, respectively. Hence the support of the term

$$
(\nabla \phi)^{2}(x)-\left(\nabla_{\gamma_{1} \circ \gamma_{2}} \phi\right)^{2}(x)
$$

is contained in $D \cup D_{a}$. Moreover, within the semi-classical approximation

$$
\chi=\chi_{c}+\text { quantum corr. }=\frac{\pi}{2 \beta}+\text { quantum corr. }
$$

and

$$
\left(\nabla_{\gamma_{1}} \chi_{c}\right)(x)=\frac{\pi}{\beta} j_{2}(x),
$$

for all $x$ in the complement of $D \cup D_{a}$. Therefore, to leading order in the semiclassical expansion,

$$
(\nabla \chi)^{2}(x)-\left(\nabla_{\gamma_{1}} \chi-\frac{\pi}{\beta} j_{2}\right)^{2}(x)
$$

vanishes, except for $x \in D \cup D_{a}$. These observations permit us to show that, to leading order in a semi-classical expansion, the right-hand side of (5.20) is nonzero. Quantum corrections are not expected to change this conclusion, (and this could actually be proven for the lattice model). Hence we expect that the scalar product

$$
\left\langle e^{-y^{0} H} \sigma\left(0, y^{1}\right) \Omega_{+}, e^{-x^{0} H} S\left(0, x^{1}\right) \Omega_{+}\right\rangle
$$

is non-zero. A slight improvement of this argument would then show that $\mathscr{H}_{\sigma}=\mathscr{H}_{s}$. Similarly, one can argue that $\mathscr{H}_{\bar{\sigma}}=\mathscr{H}_{\bar{s}}$.

4) It is easy to show that the sector $\mathscr{H}_{\bar{s}, n+1}$ and $\mathscr{H}_{s, n}$, defined in remark 2), above (5.16), coincide. Let $|s, n\rangle,|\bar{s}, n\rangle$ denote the states obtained by applying one field $s(\cdot)$ and $n$ fields $\psi(\cdot)$, for $n>0,-n$ fields $\bar{\psi}(\cdot)$, for $n<0$, to the vacuum $\Omega_{ \pm}$. One verifies easily that

$$
|\bar{s}, n+1\rangle=|s, n\rangle .
$$

Since by construction $|s, 0\rangle$ and $\langle\bar{s}, 0\rangle$ are degenerate in energy, it follows that $|\bar{s}, 1\rangle$ and $|\bar{s}, 0\rangle$ are degenerate, too. The states $|\bar{s}, 0\rangle$ and $|\bar{s}, 1\rangle$ correspond to the ones discussed by Jackiw and Rebbi [26]. 
5) Our construction of solitons with fractional fermion numbers can easily be generalized from theories with a $\mathbb{Z}_{2}$-symmetry, like the pseudo-scalar Yukawa model, to theories with a $\mathbb{Z}_{p}$-symmetry, for $p=3,4,5, \ldots$. For example, we may consider a theory of coupled Fermi fields $\psi, \bar{\psi}$ and a complex scalar field $\Phi$ with a self-interaction $P(\Phi)$, where $P$ is invariant under the transformation

$$
\Phi \rightarrow e^{2 \pi i \frac{q}{p}} \Phi, \quad q=1, \ldots, p-1 .
$$

The coupling between $\psi, \bar{\psi}$ and $\phi$ is given by the term

$$
\bar{\psi}\left(\frac{1+\gamma_{5}}{2}\right) \psi \Phi+\bar{\psi}\left(\frac{1-\gamma_{5}}{2}\right) \psi \Phi^{*},
$$

which, after bosonization, is given by

$$
: e^{i \chi}: \Phi+: e^{-i \chi}: \Phi^{*}
$$

In this model we set $m_{f}=0$. The symmetry of the model is then given by

$$
\Phi \rightarrow e^{2 \pi i \frac{q}{p}} \Phi, \quad \chi \rightarrow \chi-2 \pi \frac{q}{p},
$$

$q=0, \ldots, p-1$. For a suitable choice of the parameters, this symmetry is spontaneously broken. Using the tools developed in Sects. 2-4, one can construct soliton sectors which are eigenspaces of the fermion number operator with eigenvalue $\frac{q}{p}, q=1, \ldots, p-1$. The soliton field, $s$, of this model and the field $\phi$ satisfy the dual algebra

$$
s\left(0, x^{1}\right) \phi\left(0, y^{1}\right)=e^{\frac{2 \pi i}{p} \theta\left(y^{1}-x^{1}\right)} \phi\left(0, y^{1}\right) s\left(0, x^{1}\right) .
$$

Hence the composite field $(s \cdot \phi)(x)$ is a para-fermion field. All these claims are verified easily.

Models of the kind described here appear to be of interest in one-dimensional condensed matter physics [29].

Appendix. A C.L.H.T. Expansion for the Bosonized Yukawa Model with Thirring Term in the Lattice Approximation

In this appendix, we sketch some steps of a cluster expansion for the lattice approximation of the model (5.1) in the bosonized version.

Using this expansion, existence of soliton sectors and particle properties of the one-soliton states can be established easily, following [2, 31].

The lattice approximation of the disorder correlation functions $G_{s, \pm}\left(x_{1}, \ldots, x_{2 n}\right)[$ see Eq. (5.11)] is given by

$$
G_{s . \pm}\left(x_{1}, \ldots, x_{2 n}\right)=\frac{Z(\omega)}{Z}
$$


where $\omega_{*}$ is a $\mathbb{Z}_{2}$ lattice gauge field with $\operatorname{supp}(d \omega)=\left\{x_{i}\right\}_{i=1}^{2 n}, Z \equiv Z(\omega \equiv 1)$ and

$$
\begin{aligned}
Z(\omega)= & \int \prod d \chi_{i} d \Phi_{i} \exp -\left[\sum_{\langle i j\rangle} \frac{\beta^{\prime}}{2}\left(\chi_{i}-\omega_{i j} \chi_{j}\right)^{2}+\frac{\xi}{2} \sum_{\langle i j\rangle}\left(\Phi_{i}-\omega_{i j} \Phi_{j}\right)^{2}\right. \\
& \left.-\sum_{i} m_{f} \cos \chi_{i}-g^{\prime} \Phi_{i} \sin \chi_{i}+\frac{\lambda^{\prime}}{4}\left(\Phi_{i}^{2}-1\right)^{2}\right],
\end{aligned}
$$

and we impose 0 b.c. on $\chi-\chi_{c}, \Phi-\Phi_{c}$ at infinity. In (A.1) we have rescaled the fields $\Phi, \chi$ as

and we set

$$
\Phi \rightarrow \frac{1}{\sqrt{\xi}} \Phi, \quad \chi \rightarrow \beta \chi,
$$

$$
\beta^{\prime} \equiv \frac{1}{4 \pi \beta^{2}}, \quad \lambda^{\prime}=\lambda \xi^{2}, \quad g^{\prime}=\sqrt{\xi} g .
$$

We now decompose the basic fields $\chi$ and $\Phi$ as follows:

$$
\chi_{i}=\sigma_{i}\left(\tau_{i}+\chi_{c}+2 \pi n_{i}\right), \quad \Phi_{i}=\sigma_{i} \varrho_{i},
$$

where $\sigma_{i} \in \mathbb{Z}_{2} \simeq\{ \pm 1\}, n_{i} \in \mathbb{Z}_{+}, \tau_{i} \in\left[-\chi_{c},-\chi_{c}+2 \pi\right], \varrho_{i} \in[0,+\infty)$, and we set

$$
v_{\langle i j\rangle}=\sigma_{i} \omega_{i j} \sigma_{j} \text {. }
$$

In terms of these new variables $Z(\omega)$ reads

$$
\begin{aligned}
Z(\omega)= & \int \prod_{i} d \tau_{i} d \varrho_{i} \sum_{\left\{v_{\langle i j\rangle}\right\}} \sum_{\left\{n_{i}\right\}} \\
& \times \exp -\left(\sum_{i j} \frac{\beta^{\prime}}{2}\left[\left(\tau_{i}-v_{\langle i j\rangle} \tau_{j}\right)+2 \pi\left(n_{i}-v_{\langle i j\rangle} n_{j}\right)+\chi_{c}\left(1-v_{\langle i j\rangle}\right)\right]^{2}\right) \\
& +\sum_{\langle i j\rangle} \frac{\xi}{2}\left[\varrho_{i}-v_{\langle i j\rangle} \varrho_{j}\right]^{2} \\
& +\sum_{i}\left(\frac{-m_{f} g^{\prime}}{\left[g^{\prime 2}+m_{f}^{2}\right]^{1 / 2}} \sin \tau_{i} \varrho_{i}+\left[g^{\prime 2}+m_{f}^{2}\right]^{1 / 2}\left(1-\cos \tau_{i}\right)\right. \\
& \left.-\frac{g^{\prime 2}}{\left[g^{\prime 2}+m_{f}^{2}\right]^{1 / 2}} \cos \tau_{i} \varrho_{i}\right)+\sum_{i} \frac{\lambda^{\prime}}{4}\left(\varrho_{i}^{2}-1\right),
\end{aligned}
$$

where we have used

$$
\chi_{c}=\arctan \left(\frac{g^{\prime}}{m_{f}}\right)
$$

The Combined Low and High Temperature expansion is now constructed by treating $n$ and $v$ with low temperature methods and $\tau, \varrho-1$ with high temperature methods (see $[2,31]$ ).

The expansion converges for

large and $\beta^{\prime}$ large enough.

$$
\left|\ln \frac{\beta}{\sqrt{g^{\prime}}}\right|, \quad\left|\ln \frac{\xi}{\lambda^{\prime}}\right|, \quad\left|\ln \frac{g^{\prime}}{\sqrt{\lambda^{\prime}}}\right|
$$

In particular, it converges even for $m_{f}=0$, provided $\beta^{\prime}$ is large enough. 
Acknowledgements. We thank E. H. Lieb, J. Lott, and M. Tonin for their interest in our work and some helpful discussions.

\section{References}

1. a) Fröhlich, J.: New super-selection sectors ("soliton states") in two dimensional Bose quantum field models. Commun. Math. Phys. 47, 269 (1976)

b) Fröhlich, J.: In Current problems in elementary particle and mathematical physics. (Schladming 1976), Acta Phys. Austriaca [Suppl.] XV (1976)

c) Bellissard, J., Fröhlich, J., Gidas, B.: Soliton mass and surface tension in the $\left(\lambda|\phi|^{4}\right)_{2}$ quantum field model. Commun. Math. Phys. 60, 37 (1978)

d) Fröhlich, J.: In Invariant wave equations. Lecture Notes in Physics, Vol. 73. Berlin, Heidelberg, New York: Springer 1978

2. Fröhlich, J., Marchetti, P.A.: Soliton quantization in lattice field theories. Commun. Math. Phys. 112, 343 (1987)

3. a) Fröhlich, J.: In Recent developments in gauge theories. (Cargèse 1979) t'Hooft, G. et al. (eds.). New York: Plenum Press 1980

b) Marino, E.C., Swieca, J.A.: Order, disorder and generalized statistics. Nucl. Phys. B 170, [FS1], 175 (1980); Marino, E.C., Schroer, B., Swieca, J.A.: Euclidean functional integral approach for disorder variables and kinks. Nucl. Phys. B 200 [FS4], 473 (1982)

4. Kadanoff, L.P., Ceva, H.: Determination of an operator algebra for the two-dimensional Ising model. Phys. Rev. B 3, 3918 (1971)

Wegner, F.: Duality in generalized Ising models and phase transitions without local order parameters. J. Math. Phys. 12, 2259 (1971)

$\mathrm{t}^{\prime}$ Hooft, G.: On the phase transition towards permanent quark confinement. Nucl. Phys. B 138, 1 (1978)

5. a) Glimm, J., Jaffe, A.: Quantum physics. A functional integral point of view, 2nd edn. Berlin, Heidelberg, New York: Springer 1978

b) Glimm, J., Jaffe, A., Spencer, T.: A convergent expansion about mean field theory. I. The expansion. Ann. Phys. 101, 610 (1976)

6. Simon, B.: The $P(\phi)_{2}$ Euclidean (Quantum) field theory. Princeton, N.J.: Princeton University Press 1976

7. Osterwalder, K., Schrader, R.: Axioms for Euclidean Green's functions. Commun. Math. Phys. 31, 83 (1973); Axioms for Euclidean Green's functions. II, 42, 281 (1975)

Glaser, V.: Commun. Math. Phys. 37, 257 (1974)

8. Fröhlich, J., Osterwalder, K., Seiler, E.: Ann. Math. 118, 461 (1983)

9. Marchetti, P.A.: Constructing quantum kinks by differential geometry and statistical mechanics. To appear in Europhys. Lett., and paper in preparation

10. Constantinescu, F., Thalheimer, W.: Euclidean Green's functions for Jaffe fields. Commun. Math. Phys. 38, 299 (1974);

Seiler, E.: Gauge theories as a problem of constructive quantum field theory and statistical mechanics. Lecture Notes in Physics, Vol. 159. Berlin, Heidelberg, New York: Springer 1982

11. Köberle, R., Marino, E.C.: Duality, mass spectrum and vacuum expectation values. Phys. Lett. 126 B, 475 (1983)

12. Fröhlich, J.: Classical and quantum statistical mechanics in one and two dimensions: Twocomponent Yukawa- and Coulomb systems. Commun. Math. Phys. 47, 233 (1976)

Fröhlich, J.: In Renormalization theory. (Erice 1975) Velo, G., Wightman, A.S. (eds.), NATO Advanced Study Institutes Series C23 1976

Fröhlich, J., Park, Y.M.: Remarks on exponential interactions and the quantum sine-Gordon equation in two space-time dimensions. Helv. Phys. Acta 50, 315 (1977)

13. Brydges, D., Federbush, P.: Debye screening. Commun. Math. Phys. 73, 197 (1980)

14. Coleman, S.: Quantum sine-Gordon equation as the massive Thirring model. Phys. Rev. D 11, $2088(1975)$ 
15. Fröhlich, J., Seiler, E.: The massive Thirring-Schwinger model $\left(\mathrm{QED}_{2}\right)$ : convergence of perturbation theory and particle structure. Helv. Phys. Acta 49, 889 (1976)

16. Gallavotti, G., Nicolò, F.: The "screening phase transitions" in the two-dimensional Coulomb gas. J. Stat. Phys. 39, 133 (1985)

Nicolò, F., Renn, J., Steinmann, A.: On the massive sine-Gordon equation in all regions of collapse. Commun. Math. Phys. 105, 291 (1985)

17. Fröhlich, J., Spencer, T.: The Kosterlitz-Thouless transition in two-dimensional abelian spin systems and the Coulomb gas. Commun. Math. Phys. 81, 527 (1981)

18. Allendoerfer, C.B., Eells, J.: Commun. Math. Helv. 32, 165 (1958)

19. Bricmont, J., Fröhlich, J.: Statistical mechanical methods in particle structure analysis of lattice field theories. I. General theory. Nucl. Phys. B 251 [FS13], 517 (1985); Statistical mechanical methods in particle structure analysis of lattice field theories. II. Scalar and surface models. Commun. Math. Phys. 98, 553 (1985)

20. Artin, E.: Theorie der Zöpfe. Hamburg Abh. 4, 47 (1925)

21. Birman, J.S.: Braids, links and mapping class groups. Annals of Mathematics Studies, Vol. 82. Princeton, NJ.: Princeton University Press 1974

22. Bost, J.: In Proceedings of "VIII-th International Congress in Mathematical Physics, Mebkhout, M., Sénéor, R. (eds.). Singapore: World Scientific 1987, and references therein

23. Schroer, B., Truong, T.T.: The relativistic quantum fields of the $D=2$ Ising model. Phys. Lett. 72 B, 731 (1978); Direct construction of the quantum field operators of the $D=2$ Ising model. 73 B, 149 (1978)

24. Seiler, E., Simon, B.: Nelson's symmetry and all that in the $Y_{\text {ukawa }}$ and $\left(\phi^{4}\right)_{3}$ field theories. Ann. Phys. 97, 470 (1976)

25. Bałaban, T., Gawędzki, K.: A low temperature expansion for the pseudoscalar Yukawa model of quantum fields in two space-tıme dimensions. Ann. Inst. H. Poincaré 36 A, 271 (1982) and references therein

26. Jackiw, R., Rebbi, C.: Solitons with fermi number $\frac{1}{2}$. Phys. Rev. D 13, 3398 (1976)

27. Jackiw, R.: In Quantum structure of space and time. Duff, M.J., Isham, C.J. (eds.). London: Cambridge University Press 1982

Niemi, A.J., Semenoff, G.W.: Spectral asymmetry on an open space. Phys. Rev. D 30, 809 (1984)

28. Goldstone, J., Wilczek, F.: Fractional quantum number on solitons. Phys. Rev. Lett. 47, 986 (1981)

Frishman, R., Gepner, D., Yankielowicz, S.: Fractional charge and anomalous commutators. Phys. Lett. 130 B, 66 (1983)

29. Su, W.P., Schrieffer, J.R., Heeger, A.J.: Soliton excitations in polyacetylene. Phys. Rev. B 22, 2099 (1980)

Jackiw, R., Schrieffer, J.R.: Solitons with fermion number $\frac{1}{2}$ in condensed matter and relativistic field theories. Nucl. Phys. B 190, [FS 3], 253 (1981)

30. See e.g. Eguchi, T., Gilkey, P.B., Hanson, A.J.: Gravitation, gauge theories and differential geometry. Phys. Rep. 66, 213 (1980)

31. Marchetti, P.A.: Particle structure analysis of soliton sectors in massive lattice field theories (preprint) PD (1987)

Communicated by A. Jaffe

Received October 15, 1987 
